\title{
The Influence of Acoustic Impedance on Gaseous Layered Detonations Bounded by an Inert Gas
}

\author{
Ryan W. Houim, Robert T. Fievisohn \\ Department of Aerospace Engineering, University of Maryland, College Park, MD 20742, USA
}

\begin{abstract}
Gaseous detonations propagating through a layer of reactants that is bounded by an inert gas were simulated by solving the twodimensional reactive Euler equations. A single-step chemical reaction model was used with thermochemical properties that are representative of a highly reactive fuel-oxidizer mixture, such as stoichiometric ethylene and oxygen. A series of cases with varying acoustic impedance ratios between the inert and reactant gases, $Z$, were studied to explore the influence of acoustic impedance mismatch on the propagation of a detonation through the reactant layer. The detonation failed when $Z \sim 1$ due to a loss of triple points at the interface. The detonation propagated stably when $Z$ is high and the impedance of the inert gas is much higher than the reactants. Reflected shocks are produced from the interaction of the Mach stem of a detonation cell with the interface between the reactant and inert gases. These reflected shocks, in turn, detach and generate new triple points that are necessary to propagate the detonation. The detonation was also stable when the acoustic impedance of the inert gas is much lower than the reactants. A gas dynamic structure forms that involves a detached shock in the inert gas and a series of oblique shocks and slip lines in the reactants. A small local explosion is triggered when the Mach stem of a detonation cell interacts with the compressed reactants behind one of these oblique shocks. The resulting retonation wave produces a new Mach stem and a new triple point that leads to a stable detonation. These results suggest that the acoustic impedance and shock structure in the inert gas can have a significant influence on the stable propagation of a layered gaseous detonation.
\end{abstract}

Keywords: Detonation, Layer, Shock Wave, Acoustic Impedance, Numerical Simulation

\section{Introduction and Background}

Detonations propagating through uniform mixtures of fuel and oxidizer in confined areas have been studied extensively [1]. Relatively little attention has been given towards understanding the fundamental propagation mechanisms of layered gaseous detonations, where the reactants are semi-confined into a layer by an inert gas [2, 3]. Layered gaseous detonations have practical importance in both explosion safety and propulsion. For example, hydrogen may accumulate on the ceiling of large structures [2], high molecular weight fuels can accumulate near the ground in vapor cloud explosions [4], and detonations propagate though a layer of reactants bounded by combustion products in rotating detonation engines [5-10].

The first experiments examining detonations propagating through a layer of gaseous reactants that is bounded by an inert gas were conducted by Sommers and Morrison [11]. The general features of a gaseous layered detonation, when the acoustic impedance of the inert gas is high, are shown in Fig. 1. Here, a detonation propagates through the layer of reactants and transmits an attached oblique shock into the inert gas. This oblique shock also turns a hydrodynamically unstable contact surface between the detonation products and the inert gas.

\footnotetext{
${ }^{*}$ Corresponding Author

Email address: rhouim@umd. edu (Ryan W. Houim)
}

Sommers and Morrison [11] also developed an analytical model to calculate the angles of the transmitted oblique shock and the turned contact surface. They found that the oblique shock and contact surface angles were a function of the acoustic impedance ratio between the reactant and inert gas mixtures. An inert gas with a low acoustic impedance exhibits poor confinement and can cause the detonation to fail. An inert gas with a high acoustic impedance, however, behaves more like a solid wall and can promote a stable detonation. They also found that the transmitted oblique shock would detach for detonations propagating through a hydrogen-oxygen layer that is bounded by helium. As a result, the transmitted shock wave in the inert gas propagated ahead of the detonation. Similar detached shock structures have been observed in numerical simulations of rotating detonation engines $[5,7,9,12]$ and in the experimental work of Adams [13].

Follow-on work of gaseous layered detonations focused on the development of phenomenological models [14-18]. Some of these models were used to predict and analyze the detonation velocity deficit $[14,15]$. It was found that inert gases that have high acoustic impedance produce detonations that propagate close to the Chapman-Jouguet $(\mathrm{CJ})$ detonation velocity $\left(D_{\mathrm{CJ}}\right)$. Inert gases with a low acoustic impedance produce lower detonation velocities. Other models were developed to predict the minimum height of the reactant layer needed to support a stable detonation [16]. The detonation of gaseous reactant lay- 


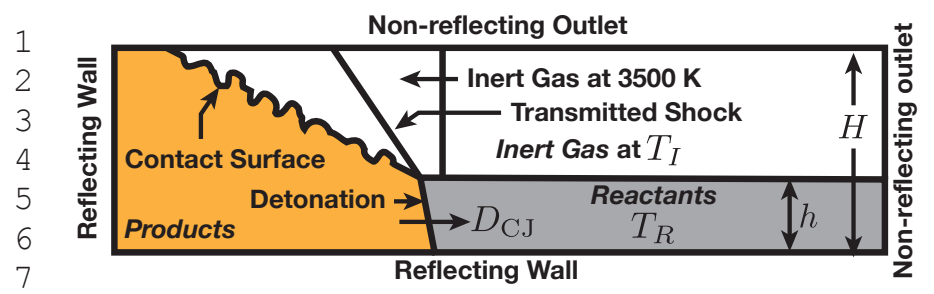

Figure 1: Initial and boundary conditions for the two-dimensional simulations of a detonation propagating through a layer of reactants bounded by an unconfined inert gas.

Gaseous interfaces do not reflect transverse shocks of detonation cells as effectively as rigid walls. In the worst case, the interaction of these transverse shocks with the surface of the reactant layer may reflect expansion waves if the acoustic impedance of the inert gas is low [28]. As a result, the critical height, $h^{*}$, for a layer of reactants that is bounded by an inert gas necessary to support a detonation is much larger than $d^{*}$. This criterion has been empirically determined to be $h^{*}>3 \lambda$ if the inert gas is confined by a solid wall [2] and $h^{*}>(12 \pm 5) \lambda$ if the inert gas is unconfined [3].

This paper presents two-dimensional numerical simulations that explore the physical mechanisms by which detonations propagate through layers of gaseous reactants bounded by an unconfined inert gas. A single-step chemical model is used with thermochemical parameters that are representative of a highly reactive fuel-oxidizer mixture, such as stoichiometric ethylene and oxygen. The influence of the acoustic impedance of the inert gas is examined in a parametric study.

\section{Model and Geometrical Setup}

The effects of viscosity and diffusion are neglected and the flow is simulated by solving reactive Euler equations,

$$
\begin{gathered}
\frac{\partial \rho}{\partial t}+\nabla \cdot(\rho \mathbf{v})=0, \\
\frac{\partial \rho Y}{\partial t}+\nabla \cdot(\rho Y \mathbf{v})=-\dot{\omega}, \\
\frac{\partial \rho \mathbf{v}}{\partial t}+\nabla \cdot(\rho \mathbf{v v})+\nabla p=0, \\
\frac{\partial \rho E}{\partial t}+\nabla \cdot[\mathbf{v}(\rho E+p)]=q \dot{\omega},
\end{gathered}
$$

where $\rho$ is the density, $\mathbf{v}$ is the velocity vector, $p$ is the pressure, $E$ is the total energy, $Y$ is the reactant mass fraction, $\dot{\omega}$ is the mass production rate of the products, and $q$ is the heat release due to chemical reaction. The gas is calorically perfect so that

$$
p=\rho R_{g} T,
$$

where $R_{g}$ is the gas constant. The molecular weight of the reactants, products, and inert gas are assumed to be the same. The total energy is

$$
\rho E=\frac{p}{\gamma-1}+\frac{1}{2} \rho \mathbf{v} \cdot \mathbf{v},
$$

where $\gamma$ is the ratio of specific heats. The sound speed is defined by

$$
c=\sqrt{\gamma p / \rho}
$$

and the acoustic impedance is

$$
\mathcal{R} \equiv \rho c=p \sqrt{\frac{\gamma}{R_{g} T}} .
$$

A single-step, irreversible, second-order chemical reaction model is adopted [29],

$$
\mathrm{R}+\mathrm{M} \rightarrow \mathrm{P}+\mathrm{M}
$$

where $\mathrm{R}, \mathrm{P}$, and $\mathrm{M}$ represent the reactants, products, and a third body, respectively. (The chaperon efficiency of the products and reactants is unity.) The mass production rate of the products is

$$
\dot{\omega}=A \rho^{2} Y \exp (-E a / R u T)
$$

where $A$ is the prefactor, $E a$ is the activation energy, and $R u$ is the universal gas constant.

The thermochemical parameters are assumed to be constant and are chosen to be representative of a highly reactive fueloxidizer mixture similar to stoichiometric ethylene and oxygen. This reaction model has been used to study deflagration-todetonation transition [30]. The thermochemical input parameters and the resulting Chapman-Jouguet (CJ) and Zeldovichvon Neumann-Döring (ZND) output detonation properties are listed in Table 1.

The governing equations are solved using the method of lines. A Strang-splitting approach is used to couple the hydrodynamic and the chemical reaction terms. A fifth-order accurate 
Table 1: Thermochemical input parameters and the output detonation properties. $D_{\mathrm{CJ}}$ is the detonation velocity, and $L_{1 / 2}$ is the half-reaction thickness of a planar detonation [30-32].

\begin{tabular}{|c|c|c|}
\hline \multicolumn{3}{|c|}{ Input } \\
\hline$\gamma$ & \multicolumn{2}{|l|}{1.2195} \\
\hline$M W$ & \multicolumn{2}{|l|}{$31 \mathrm{~kg} / \mathrm{kmol}$} \\
\hline$T_{\text {ref }}$ & \multicolumn{2}{|l|}{$298 \mathrm{~K}$} \\
\hline$E a$ & \multicolumn{2}{|l|}{$39.19 R u T_{\text {ref }}$} \\
\hline$T a$ & \multicolumn{2}{|l|}{$11,680 \mathrm{~K}$} \\
\hline$A$ & \multicolumn{2}{|c|}{$1.05 \times 10^{9} \mathrm{~m}^{3} / \mathrm{kg} \mathrm{s}$} \\
\hline$q$ & \multicolumn{2}{|c|}{$4.77 \mathrm{MJ} / \mathrm{kg}$} \\
\hline \multicolumn{3}{|c|}{ Output } \\
\hline Condition & $298 \mathrm{~K}, 1.01 \mathrm{bar}$ & $425 \mathrm{~K}, 4.56 \mathrm{bar}$ \\
\hline$D_{\mathrm{CJ}}(\mathrm{m} / \mathrm{s})$ & 2200 & 2219 \\
\hline$p_{\mathrm{ZND}}(\mathrm{bar})$ & 55 & 177 \\
\hline$p_{\mathrm{CJ}}(\mathrm{bar})$ & 28 & 90 \\
\hline$T_{\mathrm{ZND}}(\mathrm{K})$ & 1900 & 2050 \\
\hline$T_{\mathrm{CJ}}(\mathrm{K})$ & 4620 & 4760 \\
\hline$T^{*}=T a / T_{\mathrm{ZND}}$ & 6.1 & 5.7 \\
\hline$L_{1 / 2}(\mu \mathrm{m})$ & 3.2 & 0.84 \\
\hline
\end{tabular}

Godunov algorithm [33] with the HLLC approximate Riemann solver [34, 35] is used for spatial discretization. The equations are marched in time using third-order strong-stability preserving Runge-Kutta [36] with a Courant-Friedrichs-Lewy (CFL) number of 0.8 .

The chemical reaction source terms are integrated using an analytical solution by freezing the Arrhenius term during the chemical time step,

$$
\begin{aligned}
& (\rho Y)^{t+\Delta t}=(\rho Y)^{t} \exp \left(-k_{f} \Delta t\right), \\
& (\rho E)^{t+\Delta t}=(\rho E)^{t}+q\left[(\rho Y)^{t}-(\rho Y)^{t+\Delta t}\right]
\end{aligned}
$$

where $k_{f}=A \rho \exp \left(-E a / R u T^{t}\right), t$ is the solution time at the start of the chemical time step, and $\Delta t$ is the time-step size.

Adaptive mesh refinement [37] is used to dynamically refine the computational cells near detonations, shocks, and contact surfaces. The code and numerical algorithm have been extensively verified in our earlier work for many test cases including a variety of multidimensional Riemann problems, cellular detonations, flame propagation, and Richtmyer-Meshkov instabilities $[33,38]$.

Figure 1 shows the geometrical setup and boundary conditions. The height of the reactant layer, $h$, is $0.4 \mathrm{~mm}(\sim 4-5$ detonation cells) and the total height of the domain, $H$, is $6 \mathrm{~mm}$. The lower $\mathrm{x}$ - and $\mathrm{y}$-boundaries are treated as perfectly reflecting walls and the upper $\mathrm{x}$ - and $\mathrm{y}$-boundaries are assumed to be non-reflecting outlets. The initial pressure in both the reactants and the inert gas, $p_{0}$, is $1 \mathrm{~atm}$. The initial temperature of the reactants, $T_{R}$, is $300 \mathrm{~K}$.

The acoustic impedance ratio, $Z$, is defined by [11]

$$
Z \equiv \frac{\mathcal{R}_{I}}{\mathcal{R}_{R}}=\sqrt{\frac{T_{R}}{T_{I}}} .
$$

The influence $Z$ on the propagation mechanism of gaseous layered detonations was parametrically studied by varying the temperature of the inert gas, $T_{I}$. This is similar to the methods used to study shock and contact surface interactions, such as Richtmyer-Meshkov instabilities at an air and $\mathrm{SF}_{6}$ interface, using a model with constant specific heat ratio and molecular weight [39].

Acoustic impedance ratios of $1.73,1.00,0.71,0.55$, and 0.29 were considered. A summary of the cases considered and the primary results are listed in Table 1 . The $Z=1.73$ case simulates a situation where the reactants are bounded by a dense gas such as argon or $\mathrm{SF}_{6}$. The $Z=0.29$ case represents a situation where the reactant layer is bounded by high-temperature combustion products or a low-impedance gas such as helium.

The detonation for the $Z=0.29$ case was ignited with a series of $0.2 \mathrm{~mm}$-diameter hot spots of unburned material at 1500 $\mathrm{K}$ and $60 \mathrm{~atm}$. The detonation propagated for over 100 layer heights before the mechanisms of triple formation were analyzed. The detonation for the other cases were started from the stable detonation produced in the $Z=0.29$ case and abruptly changing the inert gas temperature from $3500 \mathrm{~K}$ to $T_{I}$ ahead of the detonation. This ensured that the detonations for the other cases did not fail due to a chance placement of the hot spots.

\section{Results}

\subsection{Detonation Propagation in a Channel with Solid Walls}

Figure 2 shows numerical smoke-foils for a detonation propagating in a channel of height $0.4 \mathrm{~mm}$ with rigid walls. (The numerical smoke-foils are defined by the maximum pressure in a computational cell throughout the duration of the calculation.) Three grid resolutions with minimum computation cell sizes per half-reaction thickness, $\Delta x / L_{1 / 2}$, of $4.1,8.2$, and 16.4 were examined. The results for all of the resolutions show that the detonation-cell structure is irregular with the cell size varying between $80-120 \mu \mathrm{m}$. The irregularity of the detonation cells is consistent with $T^{*}$ (see Table 2) for this reactive mixture and the results of [21] and recent experiments of expanding cylindrical $\mathrm{C}_{2} \mathrm{H}_{4}-\mathrm{O}_{2}$ detonations [40]. The average velocity of the cellular detonation was $2202 \mathrm{~m} / \mathrm{s}$ for $\Delta x / L_{1 / 2} \sim 8.2$, which is $0.1 \%$ higher than $D_{C J}$.

The bulk behavior of the detonation cells is largely the same between all of the resolutions. The grid with $\Delta x / L_{1 / 2}=8.2$ produces a slightly more irregular detonation cell structure than the coarsest grid. The finest grid shows more detail regarding the formation and merging of triple points. Nevertheless, these fine details are not the focus of this work. A detailed grid refinement study that is presented in section 4.1 shows that the bulk features and the triple formation mechanisms of the layered detonations discussed below are essentially the same on grids with $\Delta x / L_{1 / 2} \sim 8.2$ and 16.4. Thus, a grid resolution of $\Delta x / L_{1 / 2} \sim 8.2$ is used for analysis.

\subsection{Numerical Smoke-Foils}

Figure 3 shows numerical smoke-foils for all of the cases where the reactant layer is bounded by an inert gas. The smokefoil for Case 5 with a low impedance ratio $(Z=0.29 \mathrm{~K})$ shows 
Table 2: Summary of the results and parameters of a detonation propagating through a $0.4 \mathrm{~mm}$-high reactant layer that is bounded by an inert gas at temperature $T_{I}$ and an acoustic impedance ratio of $Z \equiv \mathcal{R}_{I} / \mathcal{R}_{R}$.

\begin{tabular}{cccccc}
\hline Case & $Z$ & $T_{I}$ & Detached Shock & Detonation Propagation & Initial Condition \\
\hline 0 & $\infty$ (solid wall) & - & $\infty$ & - & High-pressure pockets \\
1 & 1.73 & 100 & No & Stable & Results from Case 5 \\
2 & 1 & 300 & No & Failure & Results from Case 5 \\
3 & 0.71 & 600 & No & Failure & Results from Case 5 \\
4 & 0.55 & 1000 & Intermittent & Marginal & Results from Case 5 \\
5 & 0.29 & 3500 & Yes & Stable & High-pressure pockets \\
\hline
\end{tabular}
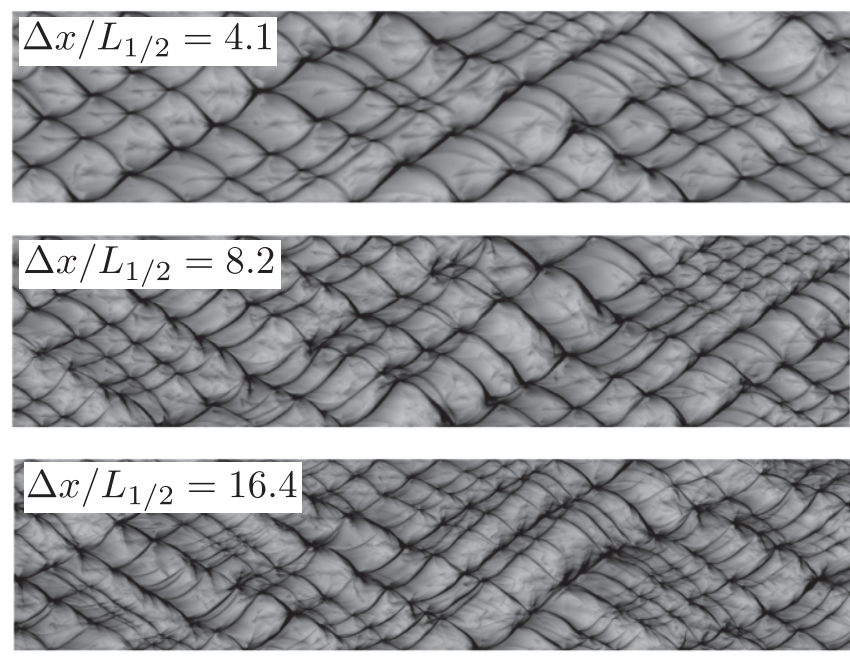

Figure 2: Numerical smoke-foils of a detonation propagating in a $0.4 \mathrm{~mm}$-high channel with solid walls using grid resolutions of 4.1, 8.2, and 16.4 grid points per half-reaction thickness. The range of $\max (p)$ lies between 30 and $300 \mathrm{~atm}$.

that a stable detonation is produced with a relatively narrow range of detonation cell sizes. The smoke-foils show that the detonations fail for Cases 2 and 3 with intermediate values of $Z$, even though they were initialized by a stable detonation. Large detonation cells and unburned fuel pockets form for Case 4, however, these unburned fuel pockets are eventually reignited and the detonation continues to propagate marginally. The detonation is stable for Case 1 where the reactant layer was confined by much a denser gas with a high acoustic impedance. The mechanisms of detonation propagation and failure for these cases are discussed below.

\subsection{Mechanism of Detonation Propagation with High Impedance Ratio (Case 1)}

Figure 4 shows the numerical schlieren $(|\nabla \rho|)$, reactant mass fraction, pressure, and temperature fields at a time of $22.6 \mu \mathrm{s}$ and time-step 120,000. (In this section, the parameter $\Delta N$ represents the number of time-steps after 120,000.) A diagram and nomenclature of the primary waves and flow structures for Case 1 are shown in Fig. 5. The bulk features of the layered detonation for this case closely resemble the attached shock structure observed in previous studies [14-16].

The overall interaction between the detonation and the interface I transmits an oblique shock into the inert gas. The oblique shock turns the flow and creates the hydrodynamically unstable contact surface between the inert gas and the products. These hydrodynamic instabilities are produced by a combination of Kelvin-Helmholtz and Richtmyer-Meshkov instabilities. The "bumps" on the oblique shock are caused by the transverse shocks of the detonation cells that periodically propagate to and strengthen local sections of the oblique shock. (See Fig. 4.)

The numerical smoke-foil in Fig. 3(a) shows that triple points propagate downwards from the interface. A time-sequence showing the details of the interaction between the transverse shock of a detonation cell with the interface is shown in Fig. 6. Each image in Fig. 6 is located in the box marked "zoom area" in Fig. 4. Initially, at $\Delta N=0$, the weak incident shock $\mathrm{S}$ of a detonation cell interacts with the interface I. This interaction transmits the oblique shock $\mathrm{O}$ into the inert gas and turns the interface to produce the material interface $\mathrm{C}$.

A detonation cell, with Mach stem M, transverse shock T, and triple point $\mathrm{A}$, propagates towards the material interfaces I and $\mathrm{C}$ at $\Delta N=0$ and 50 in Fig. 6. The transverse shock $\mathrm{T}$ and the material interface $\mathrm{C}$ interact at about $\Delta N=100$. The transverse shock impacts the material interface in an almost normal direction and produces the reflected shock R2. The reflected shock R2 is consistent with the positive acoustic impedance mismatch between the obliquely shocked inert gas and the hightemperature products [41]. The reflected shock R2 propagates down into the products and away from the leading shock of the detonation. As a result, R2 does not directly contribute to the formation of new triple points or to the stable propagation of the detonation.

The interaction between the detonation Mach stem $\mathbf{M}$ and the interface I occurs at about $\Delta N=100$. This produces the reflected shock R1 in Fig. 6, which is attached at the interface as shown in shock-polar analysis in Fig. 7 [28, 42, 43]. The reflected shock R1 is still attached to the interface and has not formed a new triple point at $\Delta N=250$. However, the detonation Mach stem $\mathrm{M}$ becomes weaker and less oblique to the interface as time increases. Eventually, the shock polars for the transmitted oblique and the reflected shocks do not intersect and a new Mach stem has formed. Consider the pressure field at $\Delta N=425$ shown in Fig. 8(a). A shock-polar analysis indicates that the polars for the transmitted oblique shock $\mathrm{O}$ and the reflected shock R1 no longer intersect. (See Fig. 8(b).) A regular refraction from the interface is impossible when these polars do not intersect and, instead, a new Mach stem is formed 


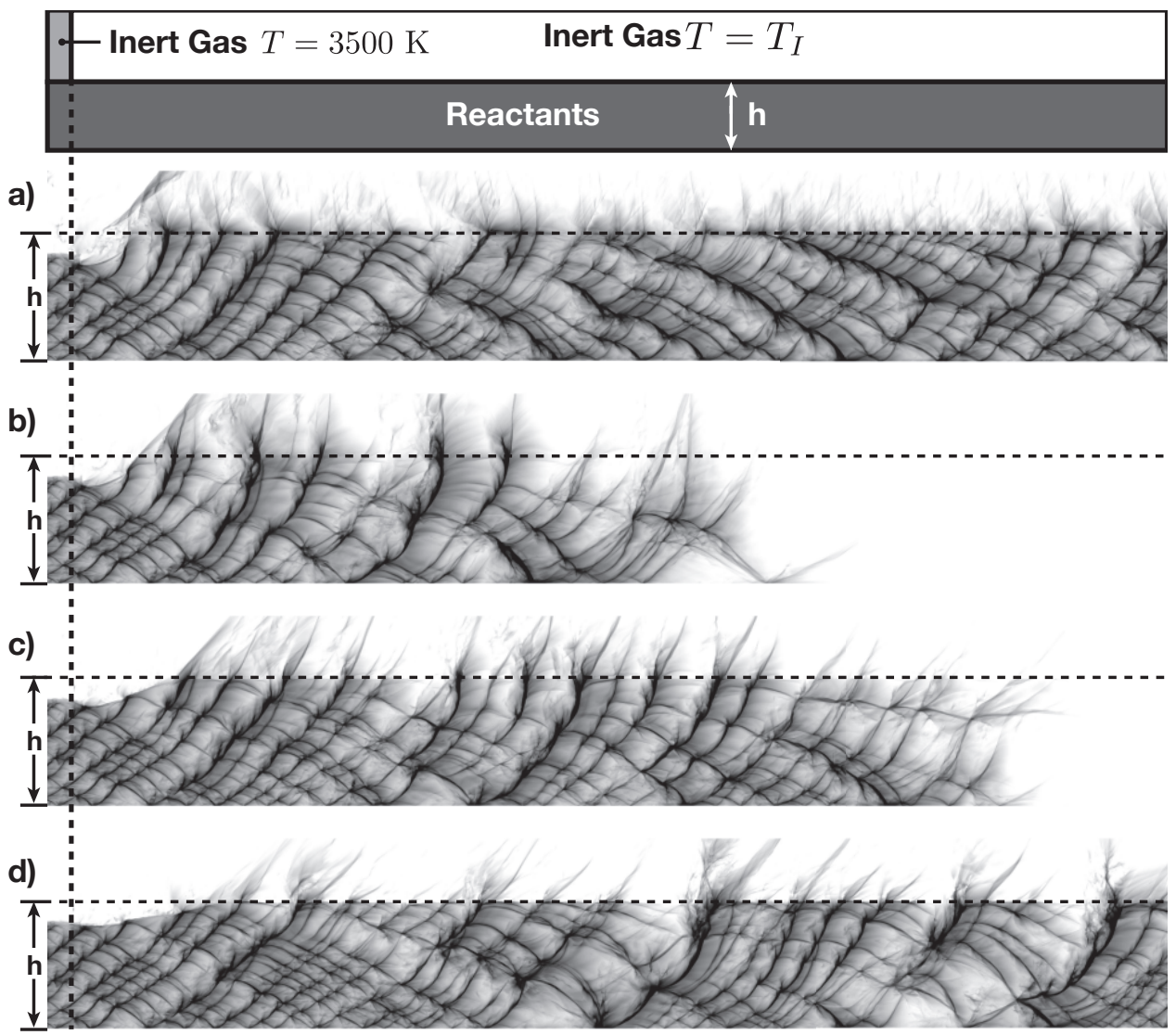

e)

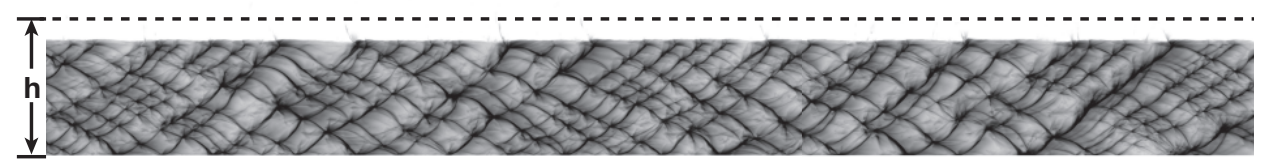

Figure 3: Numerical smoke-foil images of a detonation propagating though a layer of reactants bounded by an inert gas for (a) Case 1, (b) Case 2, (c) Case 3, (d) Case 4, and (e) Case 5. The initial height of the reactant layer is indicated by the horizontal dashed line on each smoke-foil. The location where the inert gas is transitioned from $3500 \mathrm{~K}$ to $T_{I}$ is indicated by the vertical dashed line. The range of $\max (p)$ lies between 30 and $300 \mathrm{~atm}$. 

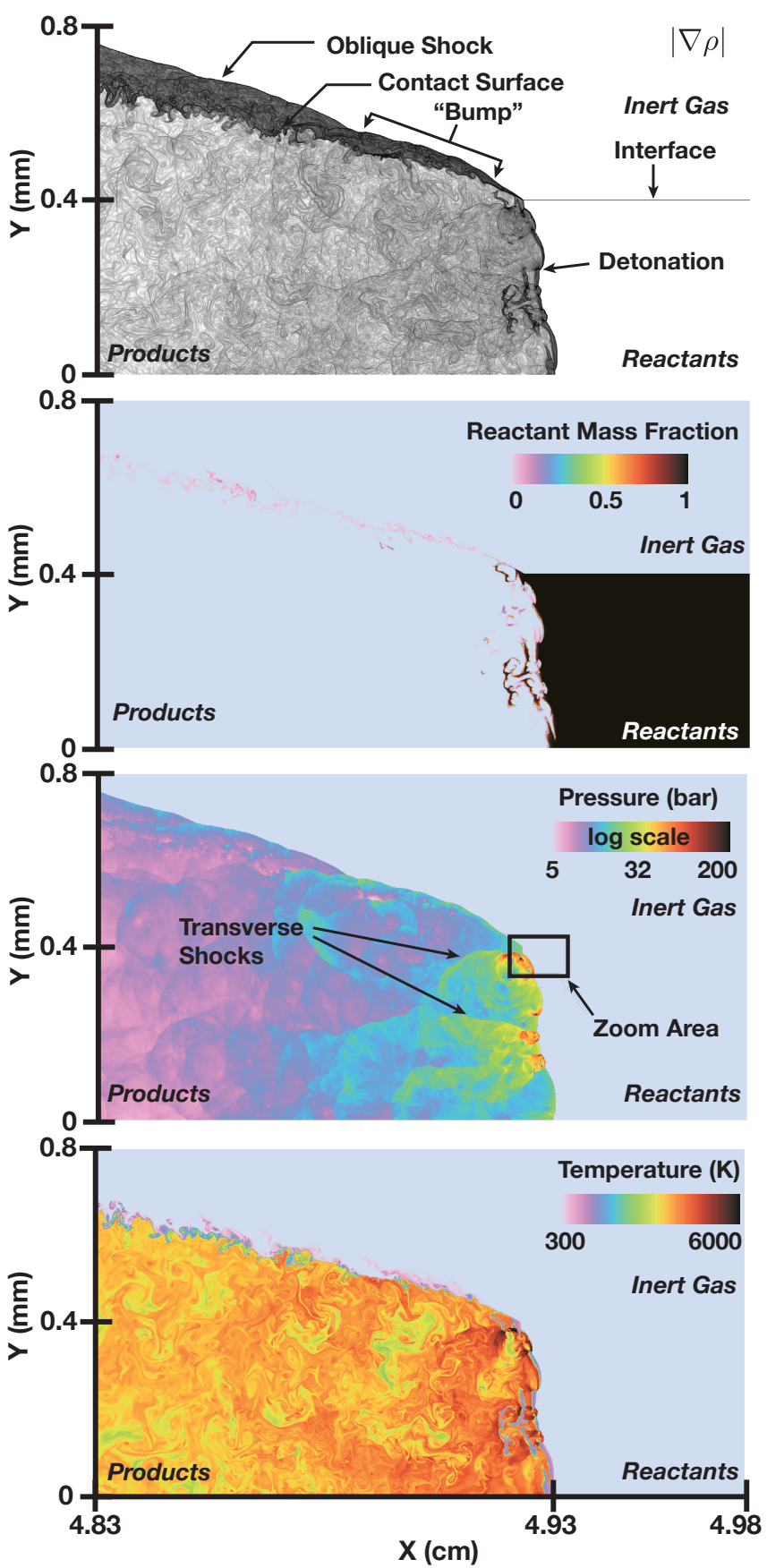

Figure 4: Color maps of the numerical schlieren $(|\nabla \rho|)$, reactant mass fraction $Y$, pressure, and temperature for Case 1 at a time of $22.6 \mu$ s and 120,000 timesteps.

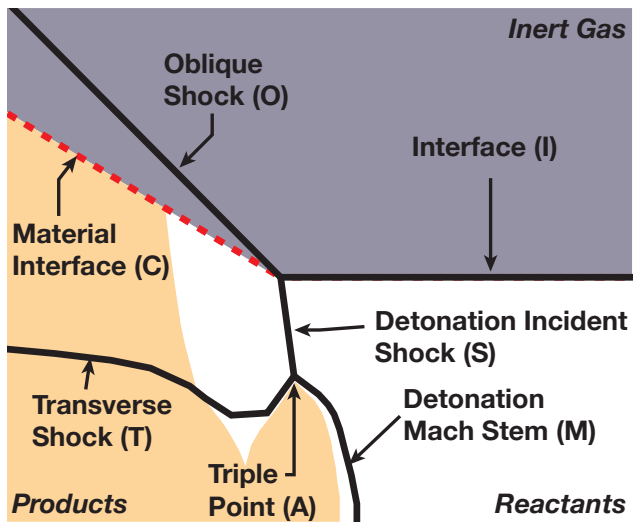

Figure 5: Sketch and nomenclature of the wave structure for Case 1 when the Mach stem of a detonation cell is about to interact with the interface between the reactant and inert gases. The thick solid lines represent the major shock waves. The red dashed line represents the material interface between the inert gas and the reactants or the products.

\section{$[28,42,43]$.}

A new detonation triple point has not yet formed at $\Delta N=$ 425, despite the new Mach stem. Figure 9 shows the process by which a new triple point is created. The pressure and burning are more intense behind the new Mach stem than they are behind the weakening lead shock $\mathrm{M}$ of the detonation cell. As a result, the velocity of the new Mach stem is about 20\% higher than the leading shock of the detonation cell. This new Mach stem catches up to $\mathrm{M}$ at $\Delta N=625$, which, in turn, causes the lead shock of the detonation to kink. (See Fig. 9c). This concave kink produces a new triple point and transverse shock. This kinking process which forms a new triple point is similar to that described in [22]. These kinks form periodically and produce a number of downward moving triple points shown in Fig. 8(a).

Not all of these newly formed triple points have equal strengths. Some of them are strong and lead to intense burning (e.g., the upper of the two downward moving triple point marked in Fig. 8(a)), while others are weak and do not significantly enhance burning. The detonation cell structure for Case 1 , as a result, is more irregular than a channel with solid walls. (Compare the numerical smoke-foils in Figs. 2 and 3(a).)

\subsection{Failure of the Detonation for with Intermediate Impedance Ratios (Cases 2 and 3)}

Figure 3(b) and (c) shows that triple points are lost when the Mach stems of the detonation cells interact with the material interface between the reactant and the inert gases for Cases 2 and 3 . There are not any reflected waves produced by this interaction for Case 2 because shock polars for the detonation Mach stem and the transmitted oblique shock completely overlap. A shock-polar analysis for Case 3 shows that the reflected wave is an expansion. (See Fig. 10.) Thus, the triple point formation mechanism discussed above for Case 1 cannot be active for Cases 2 and 3. The loss of triple points at the inert gas interface for Cases 2 and 3 is shown in Fig. 3.

The numerical smoke-foil shown in Fig. 3(b) closely resembles experimental smoke-foil images of detonations that fail 


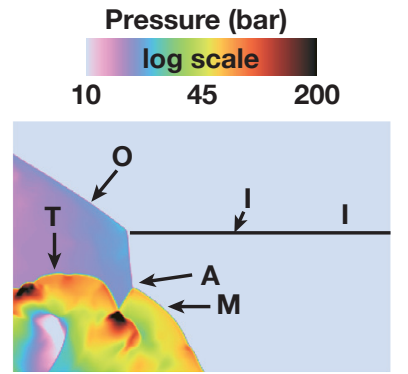

50
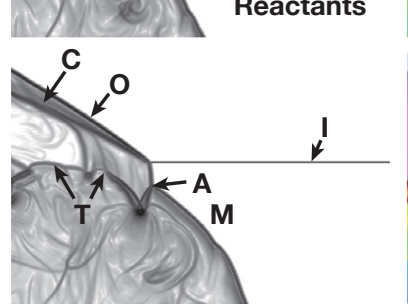

100

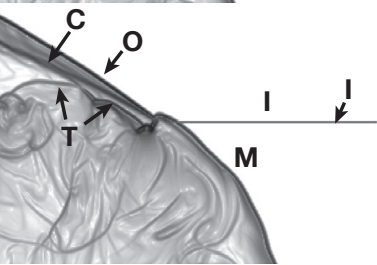

125

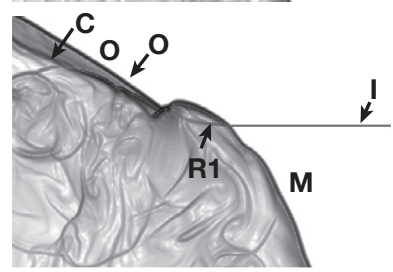

150

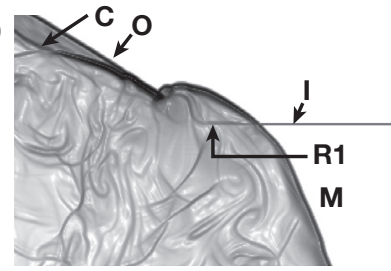

200

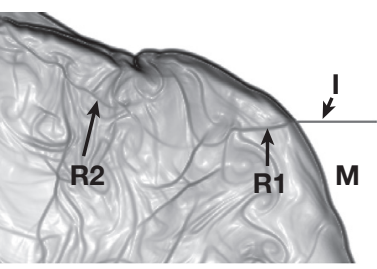

250
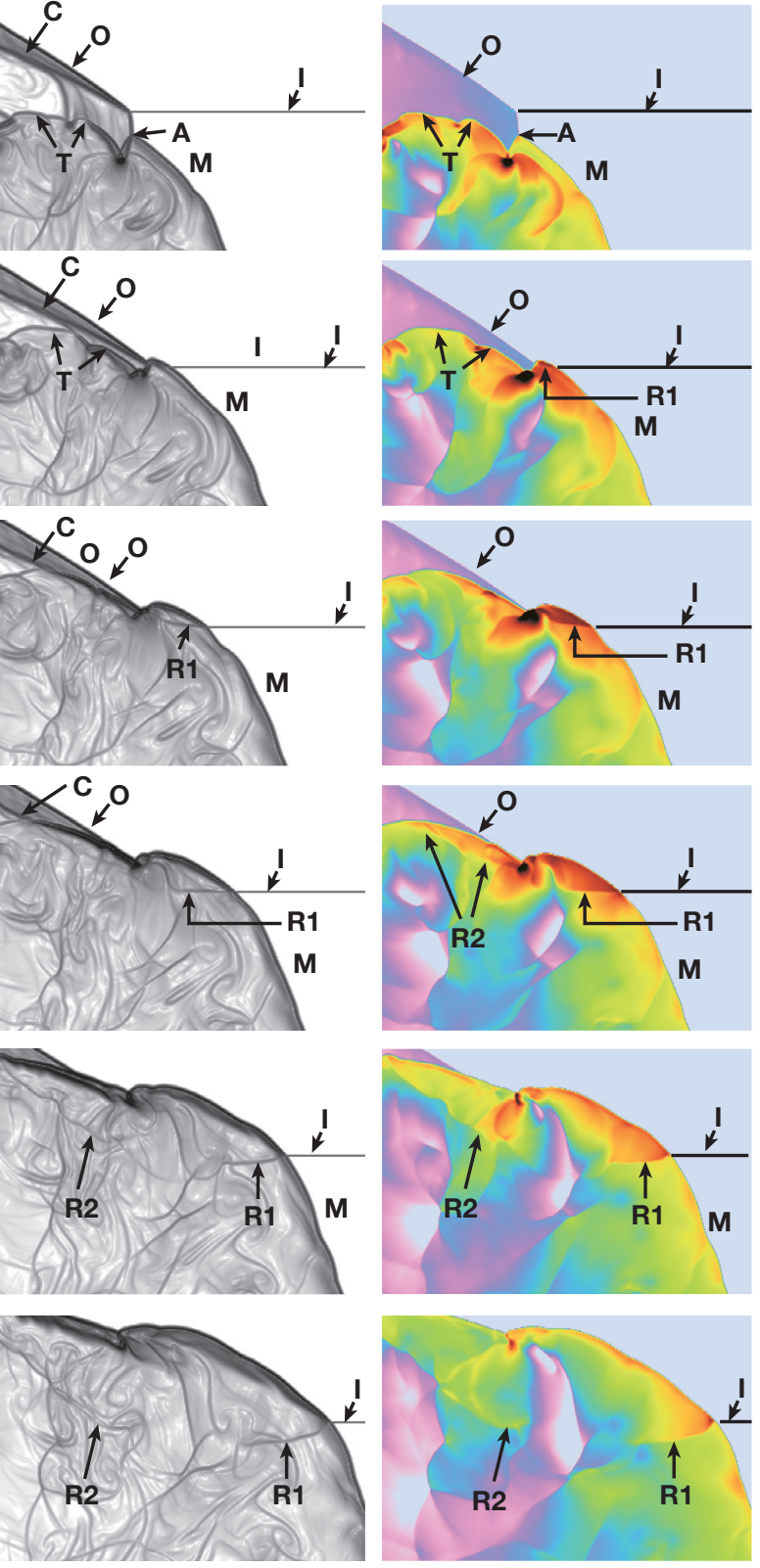

$\checkmark$
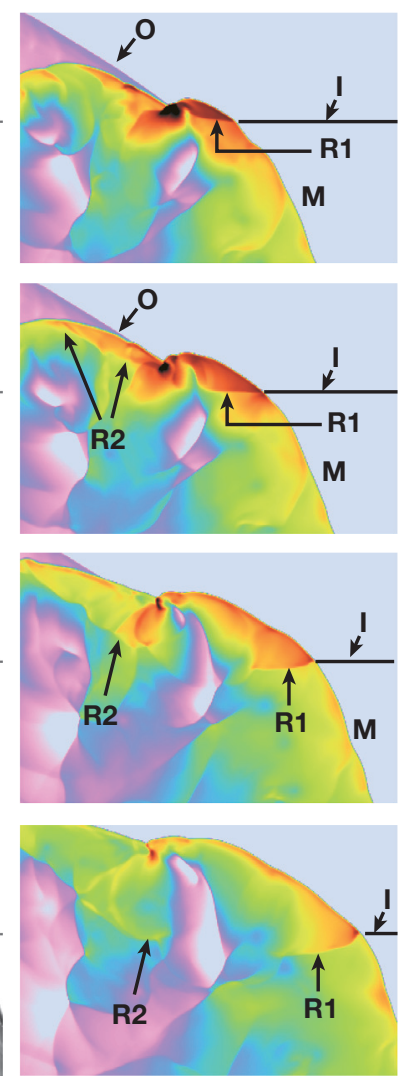

Figure 6: Color maps of the numerical schlieren $(|\nabla \rho|)$ and pressure fields in the box marked "zoom area" in Fig. 4 showing the interaction of a detonation cell with the interface for Case 1. $\Delta N$ is the number of time-steps after 120,000.

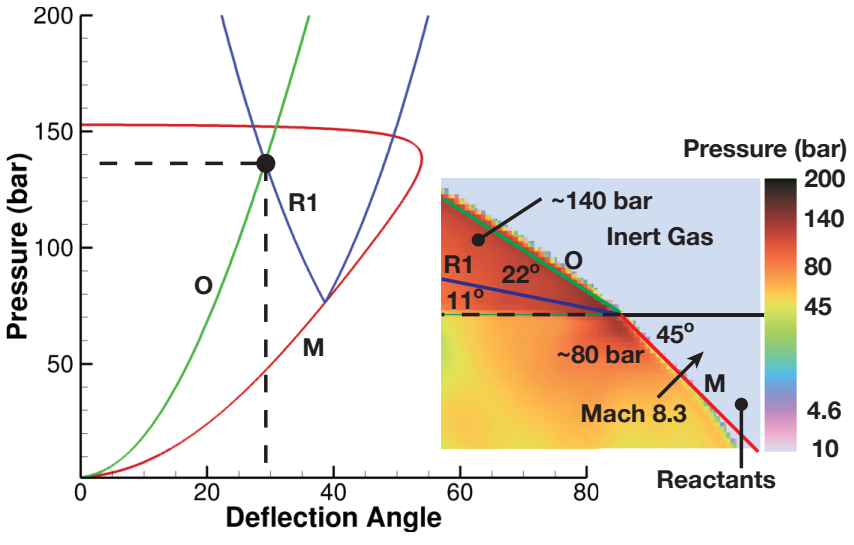

Figure 7: A shock-polar analysis of the Mach stem, M, of a detonation cell interacting with the interface between the inert and reactant gases for Case 1 at $\Delta N=150$. The Mach stem is propagating at $45^{\circ}$ with respect to the interface at Mach 8.3. The symbol $\bullet$ denotes the crossing point of the transmitted oblique shock, O, and the reflected shock, R1, polars. The inset shows a colormap of pressure close to the interface. The angles of the detached and transmitted oblique shocks are drawn and labeled based on results from the shock-polar analysis.

when propagating into a sudden expansion [19, 27, 44] or a porous tube [26]. The loss of these triple points produce large pockets of unburned material, which, in turn, cause the cells to grow until the detonation fails. The details of this failure mechanism have been discussed extensively in the literature $[19,26,27,44]$.

\subsection{Mechanism of Detonation Propagation with low Impedance Ratio (Case 5)}

Figure 11 shows the numerical schlieren $(|\nabla \rho|)$, reactant mass fraction, pressure, and temperature fields for Case 5 at $20.6 \mu \mathrm{s}$ and 102,000 time steps. ( $\Delta N$ for Case 5 refers to the number time steps after 102,000.) Figure 12 shows a diagram and nomenclature of the primary waves for Case 5. Here, in contrast to Case 1, the transmitted shock in the high-temperature inert gas is not attached, instead, it is detached and propagates ahead of the detonation. This detached shock, D, transmits the oblique shock $\mathrm{O} 1$ into the reactants and turns the interface I to produce material interface $\mathrm{C} 1$. The weak incident shock $\mathrm{S}$ of a detonation cell is a Mach stem with respect to the transmitted oblique shock O1. (This is not to be confused with the Mach stem $\mathrm{M}$ of the detonation cell.) The interaction between $\mathrm{O} 1$ and $\mathrm{S}$ produce the slip line, $\mathrm{C} 2$, and a second oblique shock, $\mathrm{O} 2$, to straighten the flow. These structures, overall, are qualitatively to the experimental schlieren images of a hydrogen-oxygen detonation bounded by pure hydrogen [13].

A shock-polar analysis shows that the structure formed by the interface I, the detached shock D, the transmitted oblique shocks $\mathrm{O} 1$ and $\mathrm{O} 2$, and the detonation incident shock $\mathrm{S}$ is a Mach lens with strong confinement [45] where there is no wave refracted into the inert gas by the detached shock. (See Fig. 13.) The shock angles and pressure behind the detached shock D and oblique shock $\mathrm{O} 1$ compare well between the shock-polar analysis and the simulation results. 


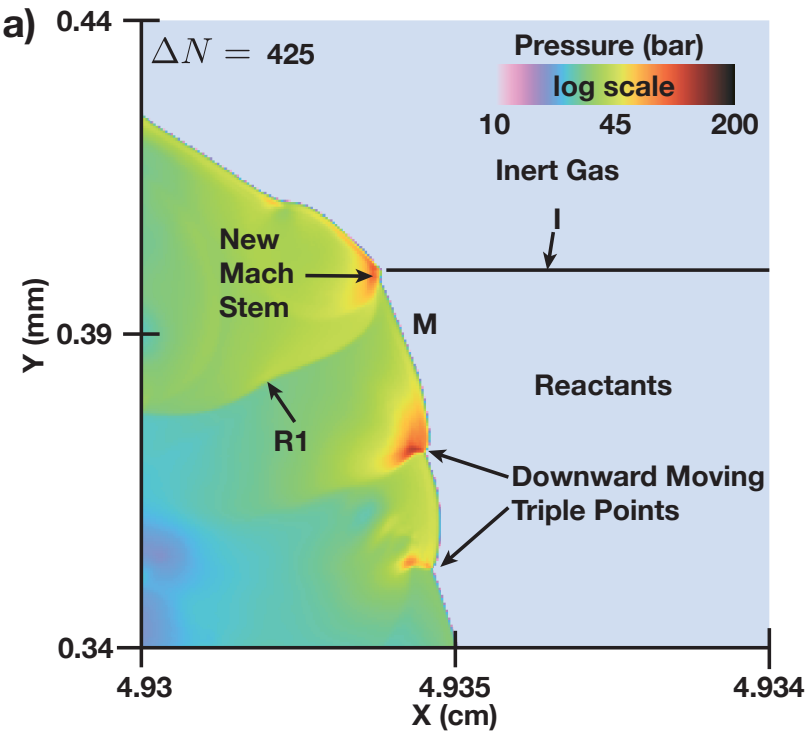

b)

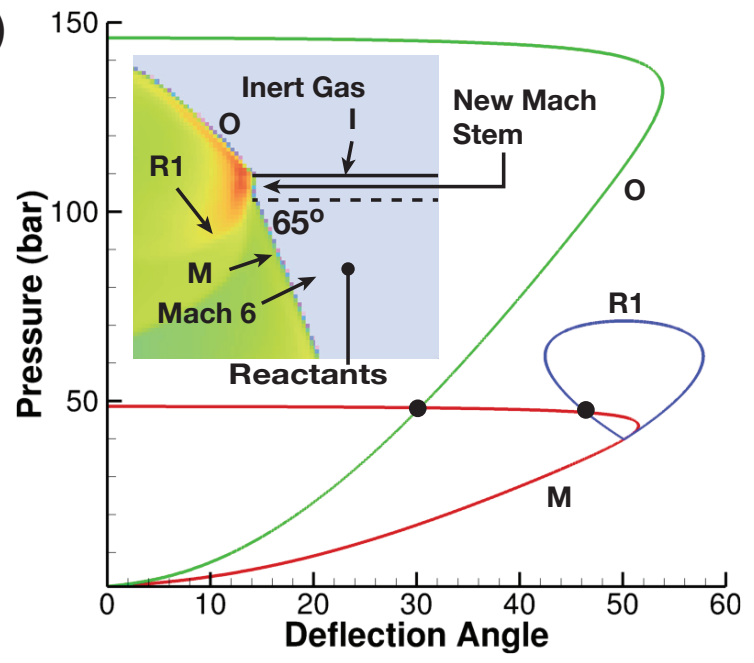

Figure 8: Plots of (a) pressure for Case 1 at $\Delta N=425$ showing multiple downward moving triple points that were generated near the interface from previous interactions between detonation Mach stems and the interface I and (b) a shock-polar analysis of the Mach stem, M, of a detonation. The Mach stem is propagating at $65^{\circ}$ with respect to the interface at Mach 6 . The symbol $\bullet$ represents the crossing points of the polars for the transmitted oblique shock, $\mathrm{O}$, and the reflected shock, R1, with the detonation Mach stem. The inset shows the pressure field near the interface.
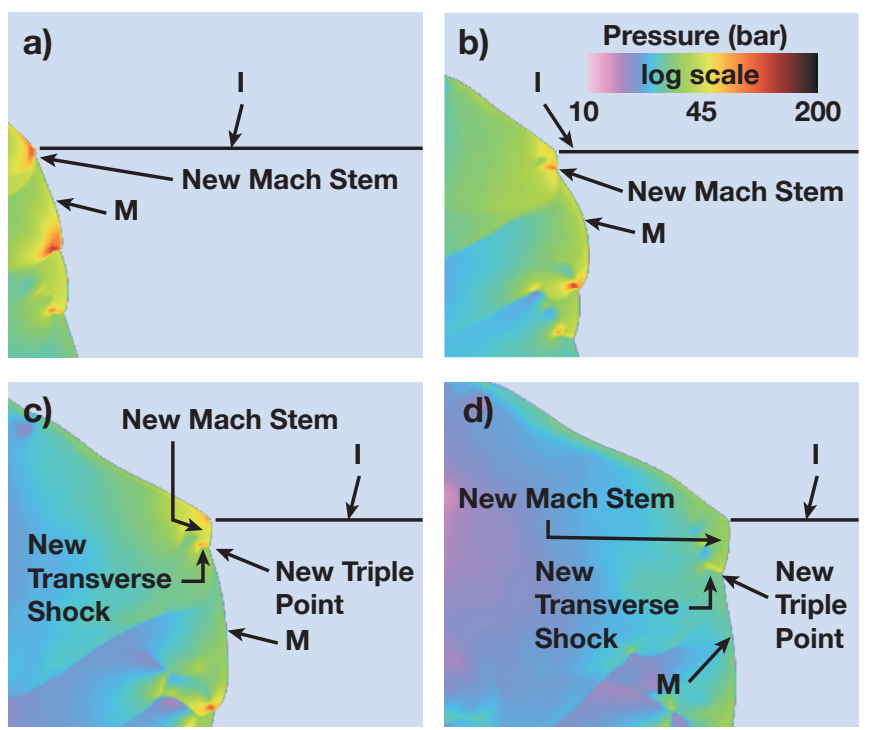

Figure 9: Time-sequence of pressure for Case 1 at later times with $\Delta N=$ (a) 425 , (b) 525 , (c) 625 , and (d) 725 showing the formation of a new triple point.

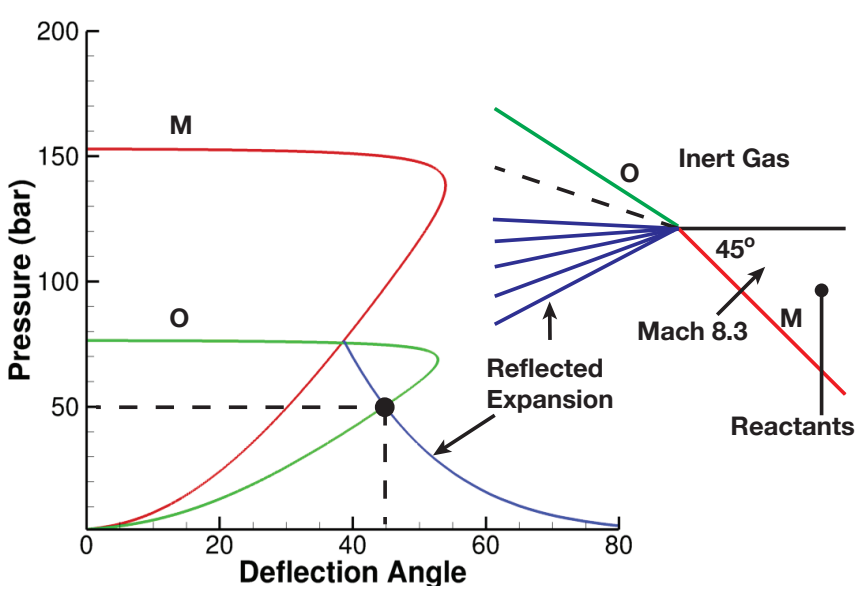

Figure 10: A shock-polar analysis of the Mach stem, M, of a detonation cell interacting with the interface I between the inert gas and the reactants for Case 3. The Mach stem is propagating at $45^{\circ}$ with respect to the interface at Mach 8.3. The symbol $\bullet$ denotes the crossing point of the transmitted oblique shock, $\mathrm{O}$, and reflected expansion wave polars. 
There is not any significant reaction near the interface prior to arrival of the detonation. Physical diffusion is not considered in this paper and there is zero numerical diffusion of the material interface I prior to arrival of the detached shock. (The HLLC approximate Riemann solver perfectly preserves a stationary contact discontinuity [35].) Weak shocks would emanate from $\mathrm{C} 1$ if there was significant reaction, but no such shocks are not observed in the numerical schlieren field shown in Fig. 11. Thus, any numerical burning near interfaces I and $\mathrm{C} 1$ is minimal and has little influence on the results.

A comparison of the smoke-foil and numerical schlieren field in Figure 14 shows that the height of the reactant layer is lowered to the point where oblique shock $\mathrm{O} 2$ and material interface C1 interact $(y \sim 0.34 \mathrm{~mm})$. Close inspection of the smoke-foil shows that the downward moving triple points are not generated at the top of the compressed reactant layer. Instead, the triple points are produced slightly beneath the surface near the location where shocks $\mathrm{O} 1, \mathrm{O} 2$, and $\mathrm{S}$ interact $(y \sim 0.32 \mathrm{~mm})$.

The time-sequence in Fig. 15 shows the interaction between a detonation cell and the Mach lens. Initially, at $\Delta N=0$ and 50 , the Mach stem $\mathrm{M}$ of a detonation cell is propagating towards the oblique shock $\mathrm{O} 1$ and the slip line $\mathrm{C} 2$. This interaction first occurs at about $\Delta N=125$. The reflected shock $\mathrm{R} 2$ is generated by the interaction of the transverse shock $\mathrm{T}$ with the slip line C2. Similar to Case 1 , the reflected shock $R 2$ is propagating away from lead shock of the detonation and does not contribute directly to the production of new triple points.

Two strong shocks, R1 and TD, are formed when the Mach stem $\mathrm{M}$ interacts with the oblique shock O1. (See Fig. 15 at $\Delta N=125$ and above.) Figure 16 shows a shock-polar analysis of this interaction at $\Delta N=150$. The velocity of the detonation Mach stem is roughly Mach 8.3 and its angle, with respect to the horizontal, is $63^{\circ}$. (This situation is modeled in the shockpolar analysis as a $17^{\circ}$ oblique shock in a $D_{\mathrm{CJ}}(\sim$ Mach 7$)$ flow interacting with a $63^{\circ}$ oblique shock in a Mach 9.3 flow. The Mach 7 and Mach 9.3 flows are separated by a slip stream.) The shock-polar analysis shows that two shock configurations are possible: a regular interaction (denoted by the $\bullet$ symbol in Fig. 16) where all of the shocks are attached, and an irregular interaction (denoted by the + symbols) $[43,46]$. The regular interaction is selected based on the simulation results. (See the pressure field in Fig. 16.) Here, unlike the previous analyses, the pressure estimated by the shock-polar analysis is significantly lower than the simulation results by $\sim 30-40$ bar. (The irregular solution would predict even lower pressure.) Thus, the strength of the reflected shocks R1 and TD cannot be explained by the reflections of two interacting shocks.

The material directly above the oblique shock $\mathrm{O} 1$ is compressed reactants at $425 \mathrm{~K}$ and 4.6 bar. The detonation velocity and ZND pressure for this compressed state are $2218 \mathrm{~m} / \mathrm{s}$ and 177 bar, respectively. (See Table 1.) The pressure behind shocks TD and R1 is much closer to the ZND pressure than the shock-polar analysis which considered both reflected shocks to be non-reactive. (See Figs. 15 and 16.) Examination of the mass fraction field (not shown) indicates that the reactants are burning behind shock TD. Thus, TD is a detonation propagating in the compressed reactants above the oblique shock O1. (See
Fig. 15 at $\Delta N=150$.) However, the detonation TD quickly fails and does not propagate very far into the compressed reactants.

The burning behind shock TD significantly strengthens and increases the pressure behind the reflected shock R1, which could be considered a retonation wave. The high strength of reflected shock R1 accelerates the process by which a new triple point is formed in comparison to Case 1. A new Mach stem (marked A in Fig. 15) is formed at $\Delta N=200$. The leading shock becomes kinked and produces a new triple point (marked B) at $\Delta N=250$. (See Fig. 15.) This new triple point is reactive and, as a result, is much stronger than the relatively unreactive triple point that was formed in Case 1 .

The triple point B2 in Fig. 15 was produced from the previous interaction between a detonation cell and the oblique shock O1. The triple point B2 strengthens and becomes part of a new detonation cell at $\Delta N=250$. This leads to a robust and stable detonation that does not contain large unburned reactant pockets.

Figure 11 shows a series of blast waves that periodically emanate from the location where $\mathrm{C} 1$ and $\mathrm{O} 2$ interact. These blast waves expand into the subsonic region behind the detached shock. They are primarily produced when the detonation TD impacts material interface $\mathrm{C} 2$. Similar blast waves are also produced by transmission of shock $\mathrm{T} 2$ through the material between contact surfaces $\mathrm{C} 2$ and $\mathrm{C} 3$. These blast waves propagate up to and reinforce the detached shock.

\subsection{Mechanism of Detonation Propagation for $Z=0.55$ (Case 4)}

The numerical smoke-foil for Case 4 indicates that the detonation is marginal. (See Fig. 3(d).) The detonation in this case has features that are similar to both Cases 3 and 5. Large pockets of unburned reactants form that are similar to those which cause the detonation to fail in Case 3. Like Case 5, however, downward-moving triple points are produced near the material interface. Some of these downward moving triple points reignite unburned material. This detonation is marginal and running the simulation for a much longer time or using a different initialization method could lead to failure.

Figure 17 shows that the detonation has a leading shock that periodically transitions between an attached structure, similar to Cases 1, 2, and 3, and a Mach lens structure, similar to Case 5. The transmitted shock is attached when a detonation cell interacts with the interface. (See Fig. 17 at $\Delta N=0,1400$, and 1700.) A weak incident shock $S$ ( Mach 4.5$)$ is produced as the Mach stem $\mathrm{M}$ of a detonation cell expands and weakens near the interface I. This incident shock decelerates more rapidly than the transmitted oblique shock in inert gas. As a result, the incident shock $\mathrm{S}$ falls behind the transmitted oblique shock becomes detached. The resulting Mach lens structure is much smaller than it is for Case 5, but similar retonation waves and triple points are produced when a detonation cell interacts with the oblique shock O1. Nevertheless, these new triple points are far weaker than they were for Case 5 and the detonation, as a result, does not propagate stably. 

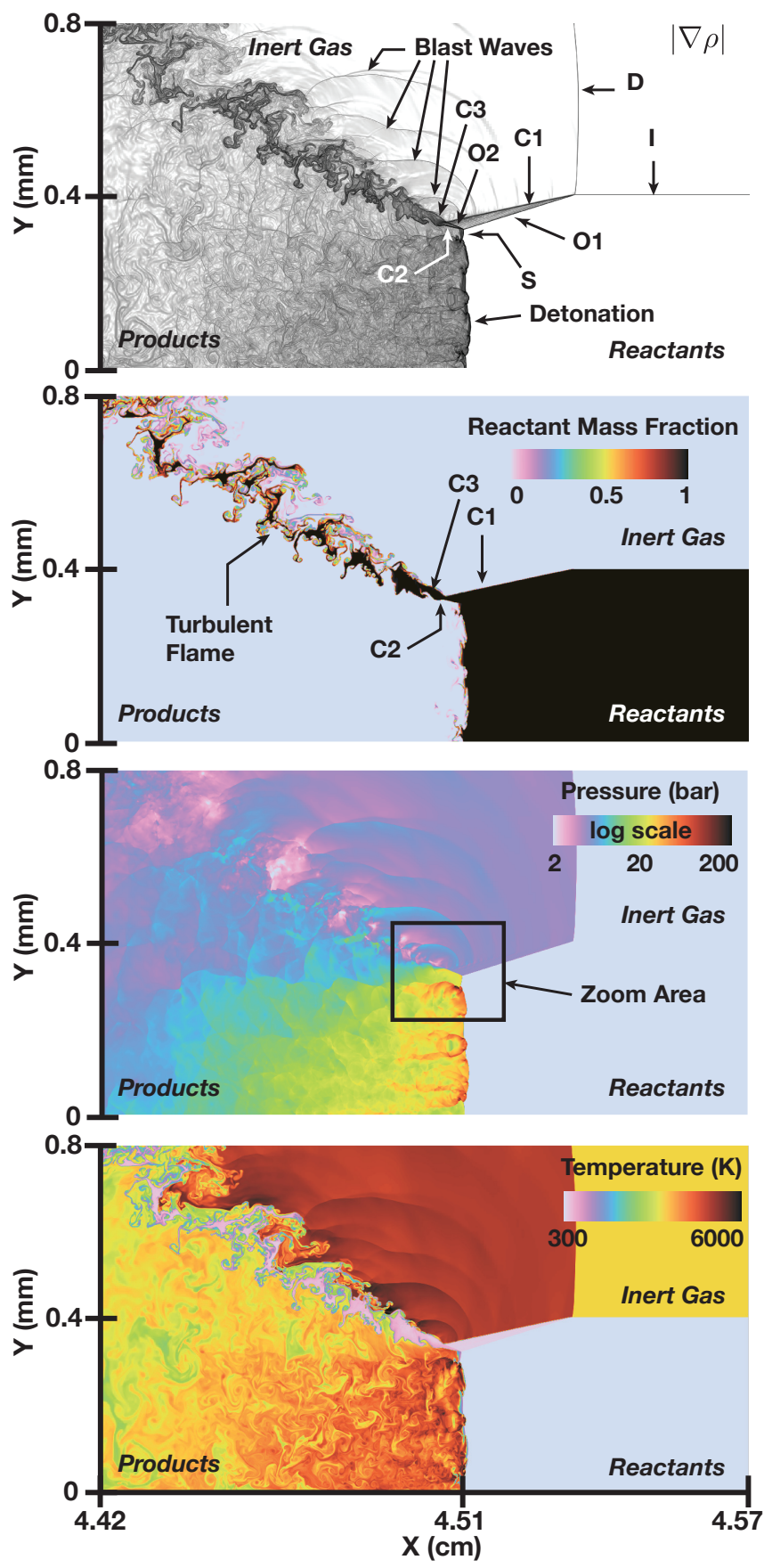

Figure 11: The numerical schlieren $(|\nabla \rho|)$, reactant mass fraction, pressure, and temperature fields for Case 5 at time step 102,000.

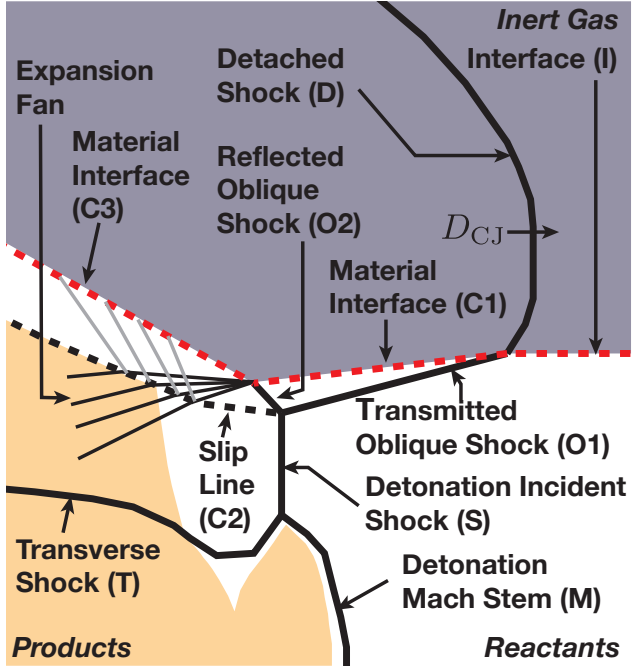

Figure 12: Sketch and nomenclature of the wave structure for Case 5. The thick solid lines are the major shock waves. The black dashed lines represent slip lines. The red dashed lines represents material interfaces between the inert gas and the reactants or products.

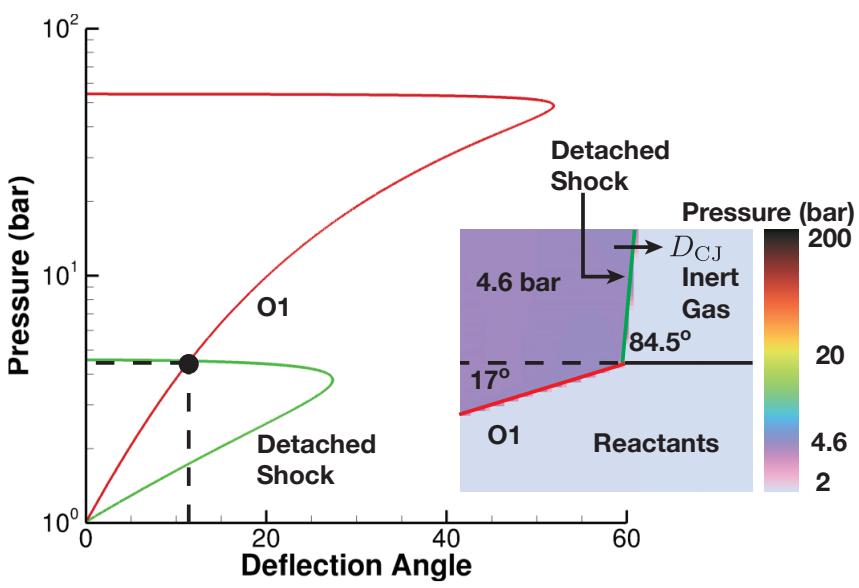

Figure 13: A shock-polar analysis of the interaction between the detached shock and the interface I for Case 5 . The detached shock propagates at $D_{\mathrm{CJ}}$. The symbol $\bullet$ denotes the crossing point of the detached and oblique shock polars. The inset shows the pressure field close to interface I. The angles of the detached shock and the transmitted oblique shock $\mathrm{O} 1$ are drawn and labeled based on results from the shock-polar analysis. 


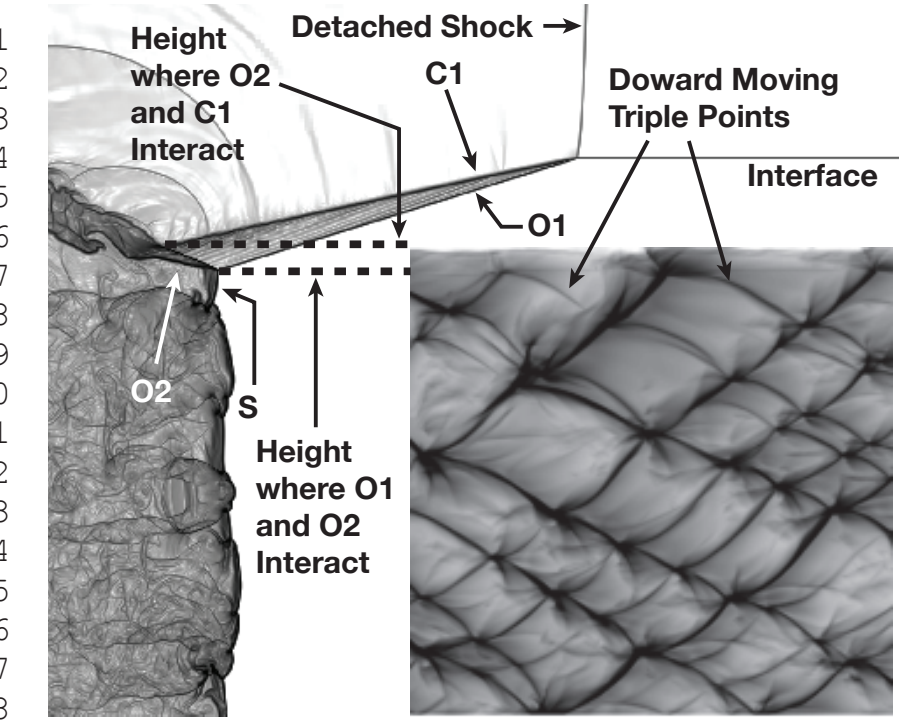

Figure 14: A close-up view of the smoke-foil and a contour map of the numerical schlieren field plotted on the same vertical scale for Case 5 .

\section{Discussion}

\subsection{Effect of Grid Refinement}

The grid resolution used in this paper, $\Delta x / L_{1 / 2}=8.2$, is often considered to be too coarse to adequately resolve irregular detonation cells. In extreme cases, well over 100 grid points per half-reaction thickness is necessary to fully resolve the detonation cells when the reactant mixture has high activation energy $[29,47]$. The simulations for Cases $1-5$ were recomputed on a finer grid with $\Delta x / L_{1 / 2}=16.4$ to check if additional grid resolution would influence the bulk features of the layered detonation and triple point generation mechanisms discussed above.

Figure 18 shows the numerical smoke-foils for Cases 1-5 on the finer grid with $\Delta x / L_{1 / 2}=16.4$. The results are similar to those on the coarser grid shown in Fig. 3. Cases 1 and 5 propagate stably and the detonation cells are qualitatively similar on both grids. Case 4 propagates marginally and produces large unburned pockets that are similar to those shown in Fig. 3. Cases 2 and 3 produce detonations that fail on the fine grid; however, their failure is faster than on the coarser grid.

The overall structure of the detonation and time sequence showing the formation of a triple point for Case 1 on the fine grid is shown in Fig. 19. The structure of the layered detonation and the mechanism by which the new triple points form are essentially the same as they were using the coarser grid as shown in Figs. 4, 6, and 9.

Figure 20 shows the overall features and triple point generation mechanism for Case 5 on the fine grid. As with Case 1, the structure of the detonation and formation of the new triple point are similar to the results on the coarse grid shown in Figs. 11 and 15. The finer grid resolves hydrodynamic instabilities (labeled I) on contact surface $\mathrm{C} 1$ as well as increased turbulence of the slip lines behind the detonation. These additional instabilities, however, do not influence the mechanism of triple point formation and the bulk features of the layered detonation.
$\Delta N$ Numerical Schlieren $|\nabla \rho|$

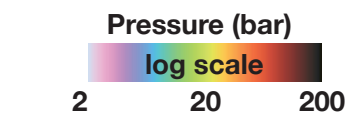

0

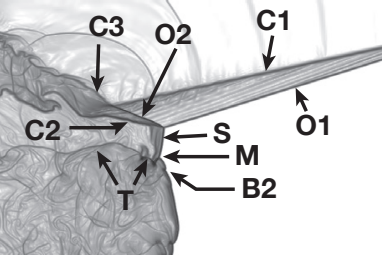

50

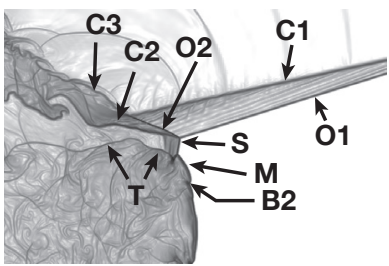

100

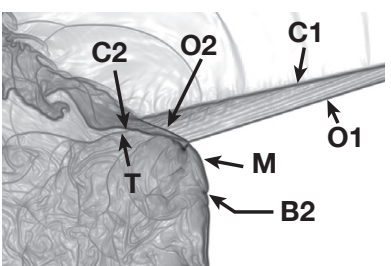

125

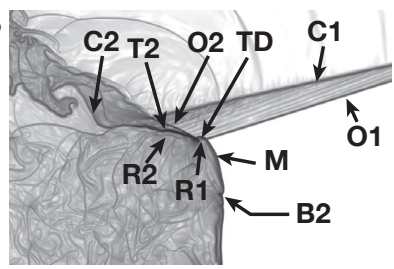

150

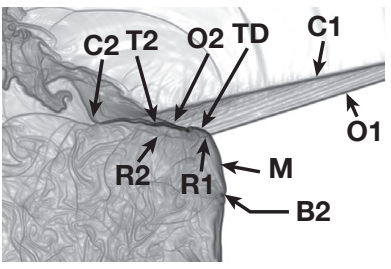

200

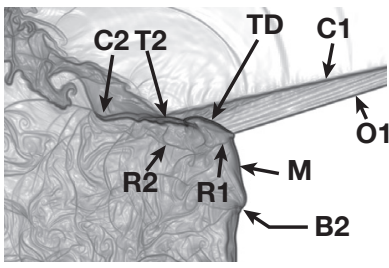

250
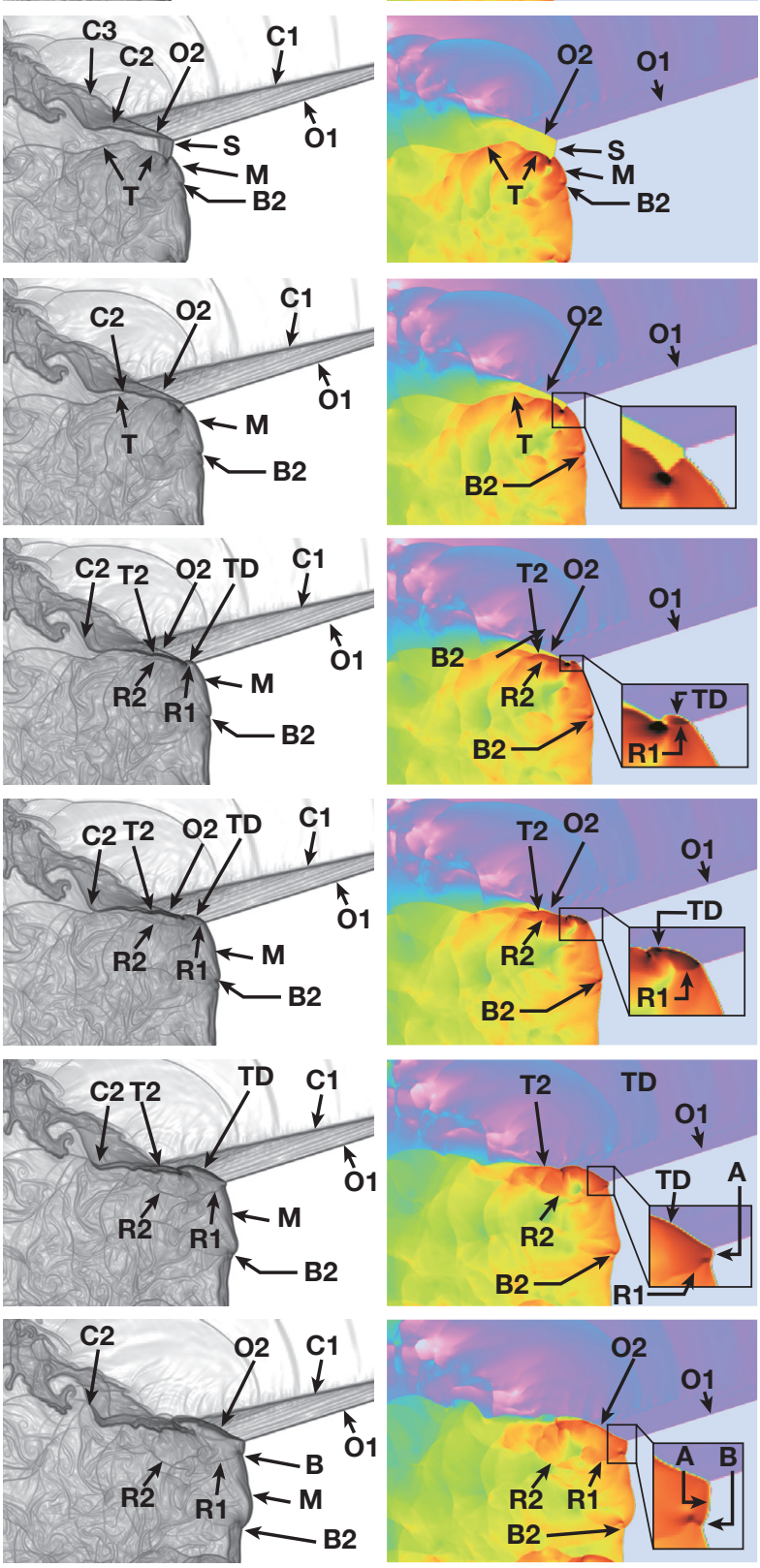

Figure 15: Contour maps of the numerical schlieren and pressure fields in the box marked "zoom area" in Fig. 11 showing the interaction of a detonation cell with the interface for Case 5. $\Delta N$ is the number of time steps after 102,000. 


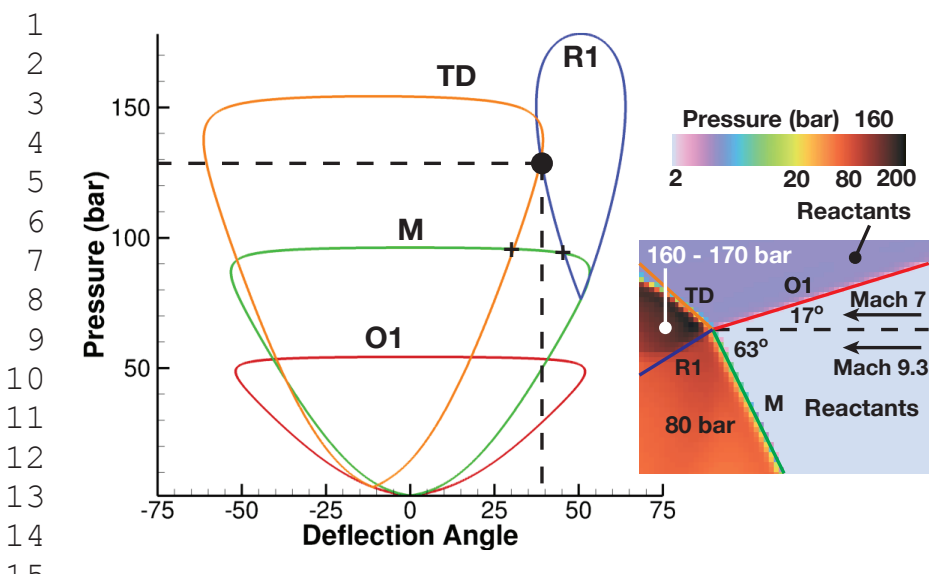

Figure 16: A shock-polar analysis of the interaction between the oblique shock $\mathrm{O} 1$ and the detonation Mach stem $\mathrm{M}$ at $\Delta N=150$ for Case 5. The symbol $\bullet$ denotes the crossing point of the shock polars for the two reflected shocks R1 and TD. The inset shows the pressure field near where shocks O1 and S interact.

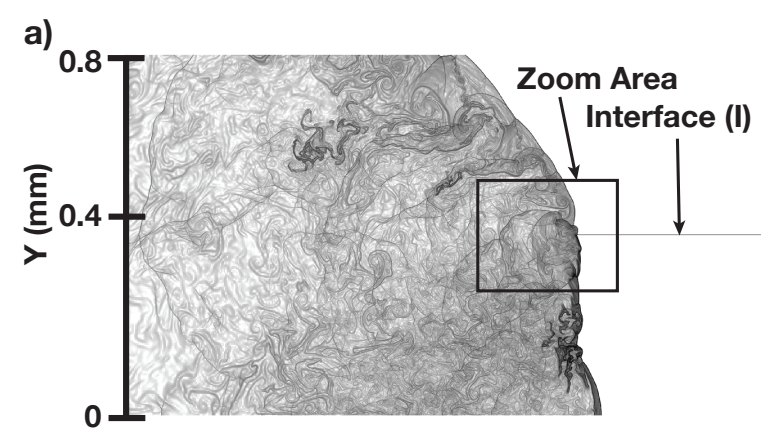

b)
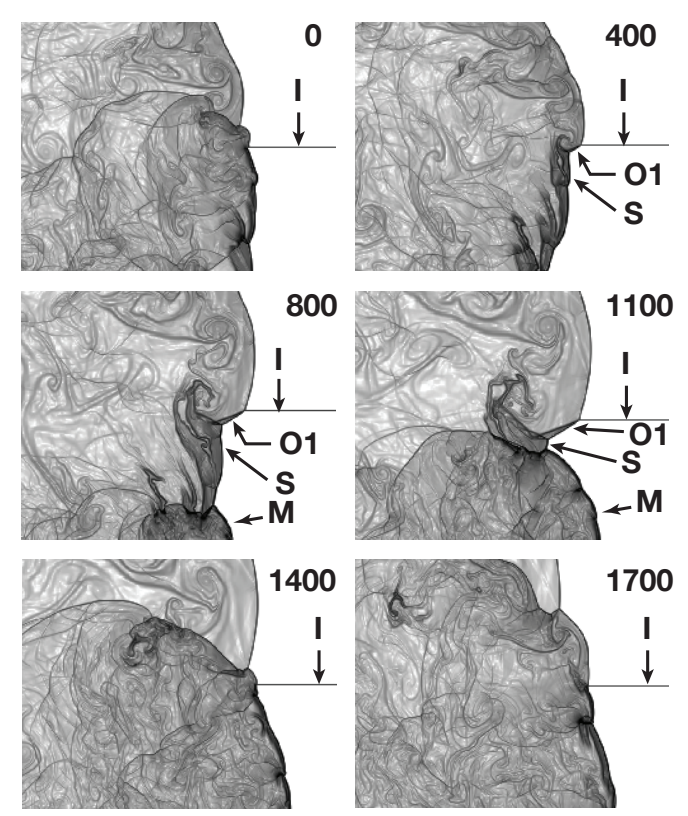

Figure 17: Numerical schlieren $(|\nabla \rho|)$ of (a) the overall detonation at time step 115,000 and (b) close-ups of the detonation near the interface at six instances of time for Case 4 . The number on the upper-right corner of each image in (b) represents the number of time- steps after 115,000 .
Table 3: Typical number of grid points for Cases 1 and 5.

\begin{tabular}{ccc}
\hline Case & $\Delta x / L_{1 / 2}=8.2$ & $\Delta x / L_{1 / 2}=16.4$ \\
\hline 1 & $6,000,000$ & $24,500,000$ \\
5 & $10,300,000$ & $47,200,000$ \\
\hline
\end{tabular}

These calculations are expensive even though adaptive mesh refinement (AMR) is used and the simulations are 2D. Table 3 lists the typical number of computational cells for Cases 1 and 5 on both grids. Case 5 sed over 45 million grid points on the finer even with AMR. Increasing the grid resolution further to $\Delta x / L_{1 / 2}=32.8$ would be computationally intensive and may require over 150 million cells for Case 5. Such fine resolution is likely unnecessary given the similarity between the smokefoils and the triple point formation mechanisms computed using grids with $\Delta x / L_{1 / 2}=8.2$ and 16.4 Nevertheless, analyzing the the fine details of the cell structure of a layered detonation or estimating the critical reactant layer height necessary to propagate the detonation would likely require a finer grid. This is left as future work.

\subsection{Consequences for Determining the Minimum Reactant Layer Height to Propagate a Detonation}

The simulation results discussed above indicate that the successful propagation of a detonation through a layer of gaseous reactants depends on many factors. The reactant layer in this work was 4-5 detonation cells high. A detonation propagating through such a thin layer should have failed according to the criterion from [3] for all of the cases. However, only Cases 2 and 3 produced failed detonations, while Cases 1 and 5 produced stable detonations. It is likely that increasing the layer height would produce a stable detonation for Cases 2 and 3. At some minimal height there would be enough upward moving transverse shocks and triple points to reignite the unburned fuel pockets. Exploring this is a topic of future study.

It is surprising that Case 5, which has the lowest acoustic impedance ratio $(Z=0.29)$, produces the most stable detonation. The high sound speed for Case 5 is responsible for producing a stable Mach lens structure. The interaction of a detonation cell with the Mach lens produces a small local explosion that, in turn, generates new triple points on the leading shock of the detonation. This process is similar to the micro-explosion mechanism discussed in [9]. Criterion of the critical reactant layer height, $h^{*}$, necessary to support a detonation need to consider the acoustic impedance of the inert gas as well as the possibility of the Mach lens structure.

\subsection{Potential Impact on Rotating Detonation Engines}

Rotating detonation engines (RDEs) are an active area of research due, in part, by their potential to significantly increase the performance and efficiency of propulsion and power generation systems [5-10]. Combustion in an RDE takes place in a combustor where a detonation continuously propagates circumferentially inside of an annular region. This detonation is sustained by continuously injecting reactants ahead of the lead 
a)

\begin{tabular}{|c|c|}
\hline - Inert Gas $T=3500 \mathrm{~K} \quad$ Inert Gas $T=T_{I}$ \\
\hline Reactants & $\uparrow \mathrm{h}$ \\
\hline
\end{tabular}

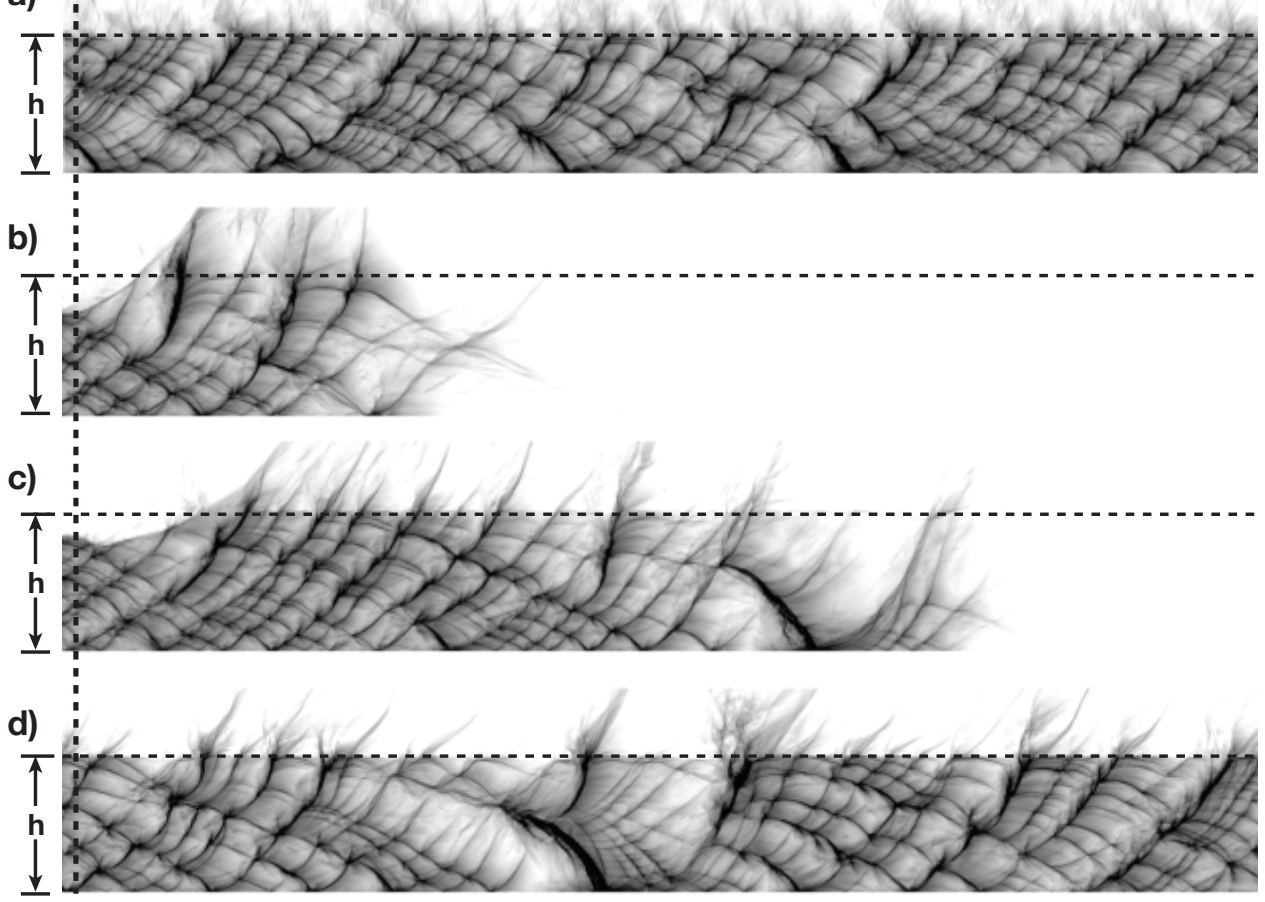

e)

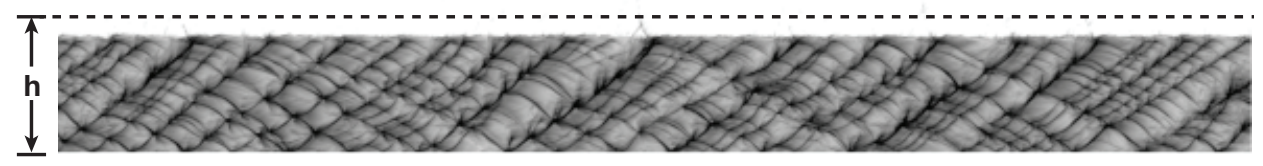

Figure 18: Numerical smoke-foil images of a detonation propagating though a layer of reactants bounded by an inert gas for (a) Case 1, (b) Case 2, (c) Case 3, (d) Case 4, and (e) Case 5. The grid resolution is $\Delta x / L_{1 / 2}=16.4$. The initial height of the reactant layer is indicated by the horizontal dashed line on each smoke-foil. The location where the inert gas is transitioned from $3500 \mathrm{~K}$ to $T_{I}$ is indicated by the vertical dashed line. The smoke-foils are defined by max $(p)$ and contours are between 30 and $300 \mathrm{~atm}$ 


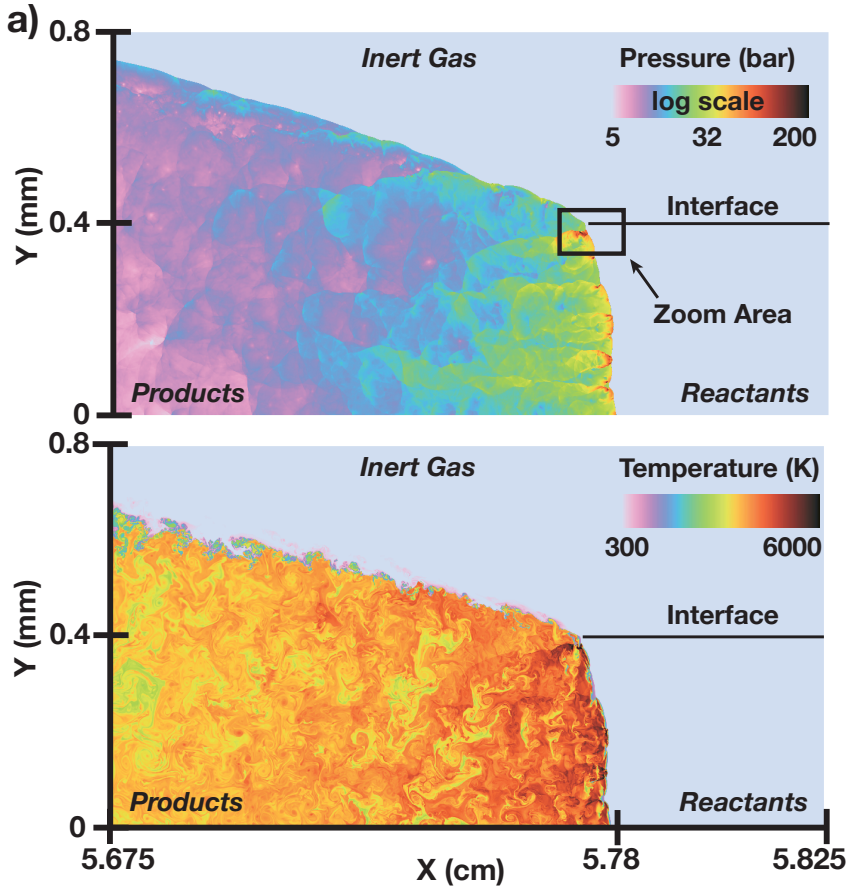

b)

$\Delta N \quad$ Numerical Schlieren $|\nabla \rho|$

0

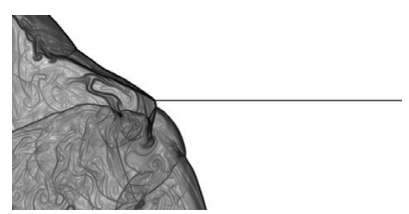

250

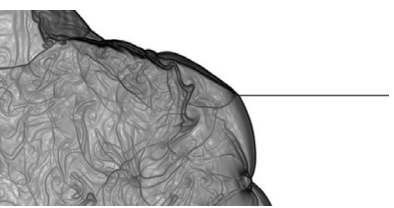

500

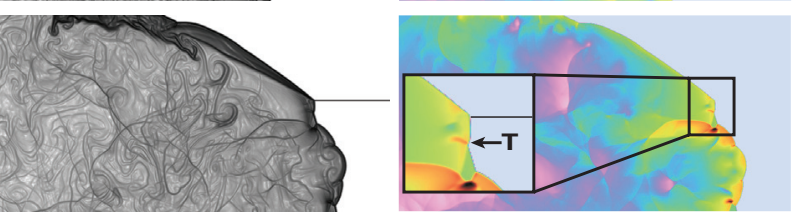

Figure 19: Results of Case 1 using $\Delta x / L_{1 / 2}=16.4$ showing (a) the overall structure of the layered detonation and (b) a time sequence showing the formation of a new triple point. The time step increment, $\Delta N$, is with respect to time step 184,500. The labels O1, R1, and T refer to the Mach stem of a detonation cell, the refracted shock from the interaction between $\mathrm{M}$ and the interface, and the new triple point, respectively.

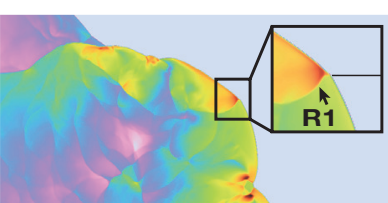

a)
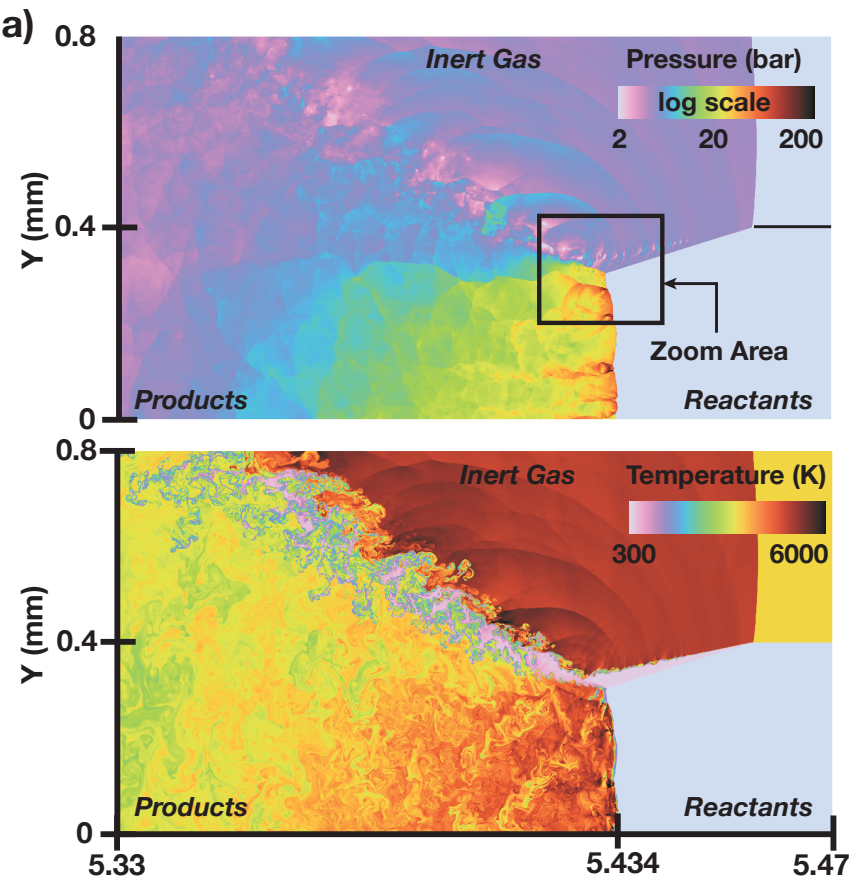

b)

$\Delta N \quad$ Numerical Schlieren $|\nabla \rho|$

0
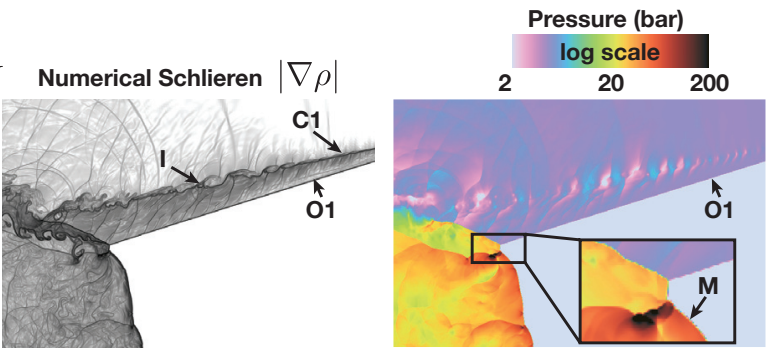

100
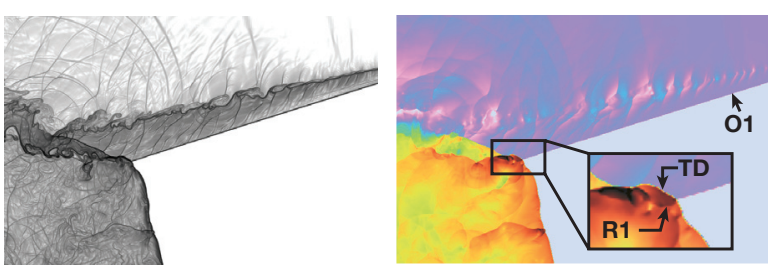

250
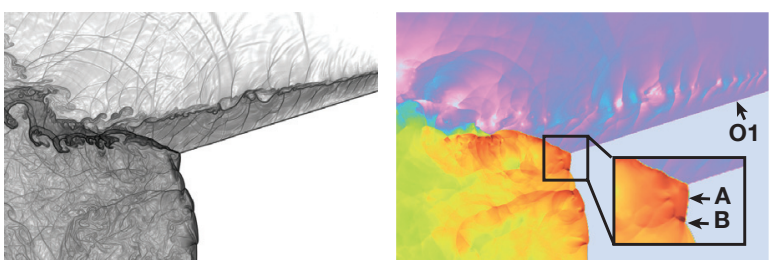

Figure 20: Results of Case 5 using $\Delta x / L_{1 / 2}=16.4$ showing (a) the overall structure of the layered detonation and (b) a time sequence of the formation of a new triple point. Here, $\Delta N$ is with respect to time step 179,600. Here M refers to the Mach stem of a detonation cell, R1 is the refracted shock from the interaction between $\mathrm{M}$ and oblique shock O1, TD is the shock of transmitted detonation behind the oblique shock, and $\mathrm{A}$ and $\mathrm{B}$ are the new Mach stem and triple point, respectively. Hydrodynamic instabilities on contact surface $\mathrm{C} 1$ are labeled I. 


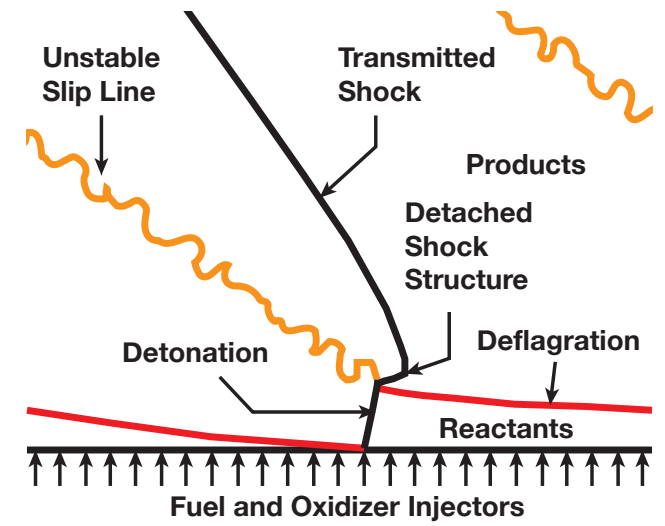

Figure 21: The main features and waves of an "unwrapped" RDE combustor based on numerical simulations $[9,12,48]$. The left and right boundaries are periodic.

shock. The high-pressure detonation products can be used to directly generate thrust in propulsion systems or spin a turbine in power generation applications. Figure 21 shows the main features and waves in an RDE combustor where the annulus has been "unwrapped".

One of the main challenges in realizing the RDE concept is to develop a basic mechanistic understanding of how detonations propagate in these devices. Figure 21 shows a detonation propagating into a reactant that is continuously being replenished by a series of injectors. The gas above the reactant layer is hightemperature combustion products produced by prior detonation waves. The products, in turn, ignite a deflagration at the contact surface separating products and reactants. The high-pressure produced by the detonation transmits an oblique shock into the products. A detached wave structure, qualitatively similar to Case 5 , is often observed in numerical simulations of RDEs $[9,12,48]$ due to the high sound speed of the products. A hydrodynamically unstable slip line forms at the contact surface that separates the products of the current detonation from the products of the previous detonation.

The combustion processes in rotating detonation engines are affected by many factors including nonuniform mixing, turbulence, wall curvature, and contact surface burning $[6,7,9]$. Nevertheless, many features of the gaseous layered detonations discussed above also pertain to RDEs. Numerical smoke-foils of a detonation propagating in an RDE show that triple points can be generated from the top of the reactant layer [7]. The inert gas above the reactant layer in an RDE consists of hightemperature combustion products and, as a result, the acoustic impedance ratio, $Z$, is low. As a result, these downward moving triple points cannot come from a regular interaction between a transverse shock and the material interface. Instead, these triple points likely originate from the interaction between a Mach lens structure formed by a detached shock and the Mach stem of a detonation cell. Simulations of detonations propagating in RDEs corroborate this and often show detached shock structures $[9,12,48]$ similar to the Mach lens structure shown in Figs. 11 and 12 .

The deflagration at the boundary between the reactants and

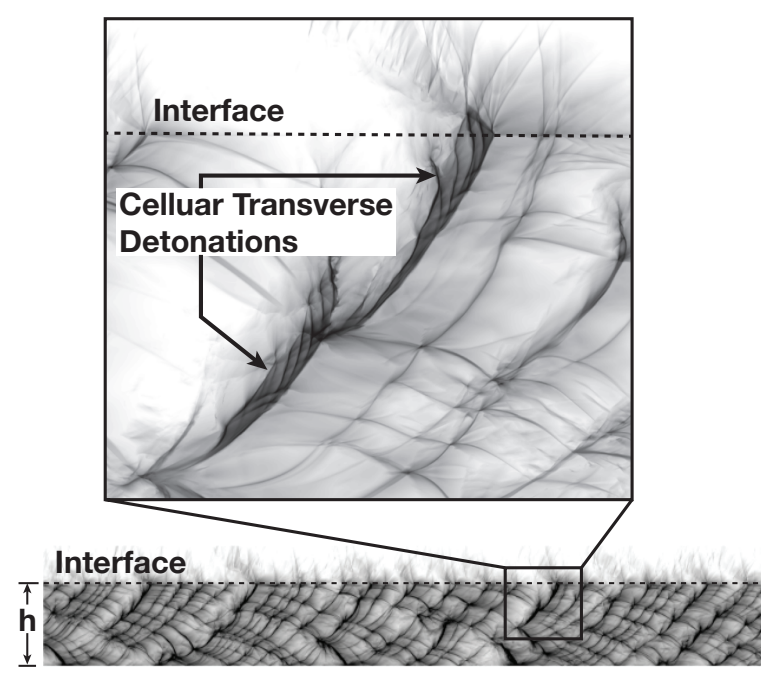

Figure 22: Numerical smokefoil records for Case 1. The zoomed region shows a cellular transverse detonation cells that interacts with the interface between the reactants and the inert gas.

products in an RDE may actually stabilize the detonation by locally decreasing $Z$ near the interface between the reactants and the products. (See the diagram in Fig. 21). The deflagration increases the temperature near the reactant and product interface to the flame temperature, which, in turn, locally decreases $Z$. This makes the detached shock structure more likely to occur. The resulting Mach lens, as discussed above, produces a stable detonation. The influence of these detached shocks to the combustion processes and stability of an RDE should now be explored in detail.

This stability of a layered detonation at low acoustic impedance ratios comes at the cost of reduced efficiency. Ideally, all of the reactants would be processed through a detonation in an RDE. Figure 11 shows that most of the reactant gas that is compressed by shocks $\mathrm{O} 1$ and $\mathrm{O} 2$ is burning as a turbulent flame for Case 5. (About $20 \%$ of the reactants would burn as a turbulent flame for Case 5 based on the initial layer height and the location where $\mathrm{O} 1$ and $\mathrm{S}$ interact.) The exact fraction of the material burning in a deflagration is difficult to determine. The transmitted detonation TD periodically propagates through the material in the left-most section of the compressed reactants. This burns some of reactant mass above oblique shock O1 as a detonation and produces the reactant gaps in the turbulent deflagration. (See. Fig. 11.) Combustion of the fuel pockets in the turbulent slip lines does not contribute to the propagation of the detonation for Case 5 .

\subsection{The Influence of Transverse Detonations}

Cellular transverse detonations occasionally interacts with the interface between the reactants and the inert gas. The smokefoil shown in Fig. 22 shows that no new triple points are made when a transverse detonation interacts with the interface. Analyzing the details of transverse detonations is a topic that should be explored as a future study. 


\subsection{Comment on Using Detailed Chemical Reaction Mecha- nisms to Simulate Layered Detonations}

The use of a simplified single-step chemical model is appropriate for this work, which is aimed at understanding the bulk features of the propagation mechanisms of layered detonations. Consider the calibration of chemical reaction mechanisms. The procedure used to calibrate the single-step ethyleneoxygen model used in this paper is the same as that described in [29]. The activation energy, pre-exponential factor, specific heat ratio, chemical energy, etc. are optimized to match data on laminar flame thickness and speed, adiabatic flame temperature, detonation velocity, and detonation half-reaction thickness, etc. Thus, the single-step reaction mechanism used in this work is calibrated using data for detonations.

Figure 21 of Oran [49], however, shows that there is very little overlap in the pressure-temperature space relevant to hydrogen-air detonations and the data used to calibrate detailed chemical reaction mechanisms for hydrogen. The preexponential factors, temperature exponents, activation energies, and pressure falloff parameters for detailed chemical reaction mechanisms are usually calibrated for data on flames, ignition delay times in shock tubes, etc. The pressures and temperatures relevant to detonations are usually outside of the calibration regimes for reaction rate parameters. Detailed chemical mechanisms, as a result, need to be extrapolated significantly beyond their calibration regime when used to simulate cellular detonations.

It has been shown that detailed chemical reaction mechanisms fail to produce the experimental size and regularity of detonation cells for mixtures of hydrogen and air at atmospheric pressure and temperature [50]. It was proposed that thermodynamic nonequilibrium behind the strong leading shock wave of detonation may be important for detonations. Thermodynamic nonequilibrium, however, is not taken into account by detailed chemical kinetics and could be a physical reason why the computed detonation cells have poor agreement with experimental results. The effect of thermodynamic nonequilibrium on detonations, could, in principle, be implicitly taken into account if a reaction mechanism is calibrated for detonations. However, this is not the case for most detailed chemical reaction mechanisms. The bulk influence of thermodynamic nonequilibrium, if it is important, is, in principle, implicitly accounted for during the calibration of the single-step reaction mechanism and gas-dynamic parameters.

\section{Summary and Conclusions}

This paper presented two-dimensional numerical simulations of a cellular detonation propagating through a layer of gaseous reactants that is bounded by an inert gas. A simplified singlestep chemical reaction model is used to describe the mass consumption rate of the reactants. The thermochemical parameters are selected to be representative of a highly reactive fuel and oxidizer mixture similar to stoichiometric ethylene and oxygen. The influence of the acoustic impedance ratio between the inert and reactant gases $(Z)$ on the mechanism of detonation propagation was examined through a parametric study. Cases where $Z$ was high (simulating a dense inert gas such as $\mathrm{Ar}$ or $\mathrm{SF}_{6}$ ), where $Z$ was low (simulating a light inert gas such as He or combustion products, and where $Z$ was intermediate were studied. The height of the reactant layer was $0.4 \mathrm{~mm}(\sim 4-5$ detonation cells $)$ in all of the cases examined.

The detonation fails when $Z \sim 1$. This is consistent with the minimal height criterion of Bykovskii et al. [3]. A shock-polar analysis showed that either no wave or an expansion wave is refracted when a Mach stem of a detonation cell interacts with the interface between the inert gas and the reactants when $Z \sim 1$. The transverse shocks and triple points necessary to produce a stable detonation are not generated. Instead, triple points of the detonation are lost at the interface and, as a result, the detonation cells grow until the detonation fails. It is likely that increasing the layer height would eventually produce a stable detonation. Exploring this is left for future work.

The detonation propagates stably when the inert gas is much more dense than the reactants $(Z=1.73)$. A shock wave is refracted when the Mach stem of a detonation cell interacts with the interface between the dense inert gas and the reactants. This reflected shock is initially attached to the interface, but detaches and forms a new Mach stem as the detonation cell expands and weakens. The burning behind this new Mach stem is more intense than it is behind the remnant of the detonation cell. The new Mach stem, as a result, moves faster and catches up to the old detonation cell. This, in turn, kinks the lead shock of the detonation and produces a new triple point. Some of these new triple points are strong and promote vigorous combustion while others are weak and essentially nonreactive. Nevertheless, the detonation propagates stably.

The detonation is also stable when acoustic impedance ratio is low $(Z=0.29)$. The sound speed of the inert gas is high enough that the shock wave that is transmitted into the inert gas detaches and propagates in front of the detonation. This detached shock, in turn, transmits an oblique shock into the reactants, which interacts with the weak incident shock of the detonation. Overall, this shock structure resembles a Mach lens [45]. New triple points are produced when the Mach stem of a detonation cell interacts with the transmitted oblique shock. This interaction triggers a small explosion in the compressed reactants above the oblique shock. The resulting retonation wave directly produces a new Mach stem and triple point on the leading shock of the detonation. Most of these new triple points are reactive. The detonation, as a result, is very stable even though an acoustic impedance or shock-polar analysis at the interface would predict reflected expansion waves.

The detonation propagates marginally when $Z=0.55$. Here, the acoustic impedance ratio is between the limits that produce the Mach lens and rapid failure. The Mach lens structure, which is largely responsible for creating a robust mechanism for generating transverse waves for the low- $Z$ case, appears and disappears periodically. Triple points are generated when the Mach lens is present. Nevertheless, these new triple points are much weaker than they are for the the low- $Z$ case. The detonation in case is not robust and propagates marginally.

Correlations and analytical models developed to estimate the minimum reactant layer height necessary to promote a stable 
detonation must consider the possibility of the detached Mach lens shock structure. The high temperature of the detonation and deflagration products in a rotating detonation engine may actually promote the formation of the detached shock structure and, in turn, possibly enhance the boundaries of the stable operating conditions. Exploring the effect of layer height, igniting a deflagration at the interface between the inert gas and the reactants, and including realistic temperature profiles in the inert gas that are consistent with RDEs is left for a future study.

\section{Acknowledgments}

The authors thank Elaine Oran, Kenneth Yu, and Jason Burr for their thoughtful discussions on detonations propagating in layers and in rotating detonation engines. This work was supported in part by the National Science Foundation Graduate Research Fellowship Program under Grant No. DGE 1322106 and in part by the University of Maryland through Minta Martin Endowment Funds in the Department of Aerospace Engineering, and through the Glenn L. Martin Institute Chaired Professorship at the A. James Clark School of Engineering. The authors acknowledge the DoD High-Performance Computing Modernization Program and University of Maryland supercomputing resources (http://www.it.umd.edu/hpcc) made available in conducting the research reported in this paper.

\section{References}

[1] J. H. Lee, Dynamics parameters of gaseous detonations, Annual Review of Fluid Mechanics 16 (1984) 311-336.

[2] W. Rudy, M. Kuznetsov, R. Porowski, A. Teodorczyk, J. Grune, K. Sempert, Critical conditions of hydrogen-air detonation in partially confined geometry, Proceedings of the Combustion Institute 34 (2) (2013) 1965 1972.

[3] F. A. Bykovskii, S. A. Zhdan, E. F. Vedernikov, Continuous spin detonations, Journal of Propulsion and Power 22 (6) (2006) 1204-1216.

[4] D. M. Johnson, The potential for vapour cloud explosions - lessons from the buncefield accident, Journal of Loss Prevention in the Process Industries 23 (6) (2010) $921-927$.

[5] P. Wolaǹski, Detonative propulsion, Proceedings of the Combustion Institute 34 (1) (2013) 125 - 158.

[6] F. K. Lu, E. M. Braun, Rotating detonation wave propulsion: experimental challenges, modeling, and engine concepts, Journal of Propulsion and Power 30 (5) (2014) 1125-1142.

[7] M. Hishida, T. Fujiwara, P. Wolanski, Fundamentals of rotating detonations, Shock Waves 19 (2009) 1-10.

[8] K. Kailasanath, Review of propulsion applications of detonation waves, AIAA Journal 38 (9) (200) 1698-1708.

[9] Y. Uemura, A. K. Hayashi, M. Asahara, N. Tsuboi, E. Yamada, Transverse wave generation mechanism in rotating detonation, Proceedings of the Combustion Institute 34 (2013) 1981-1989.

[10] B. Voitsekhovskii, Maintained detonations, in: Soviet Physics Doklady, Vol. 4, 1960, p. 1207.

[11] W. P. Sommers, R. B. Morrison, Simulation of condensed-explosive detonation phenomena with gases, The Physics of Fluids 5 (2) (1962) 241248.

[12] D. Schwer, K. Kailasanath, Fluid dynamics of rotating deotnation engines with hydrogen and hydrocarbon fuels, Proceedings of the Combustion Institute 34 (2013) 1991-1998.

[13] T. G. Adams, Do weak detonations exist, AIAA Journal 16 (10) (1978) 1035-1040.

[14] E. K. Dabora, J. Nicholls, R. Morrison, The influence of a compressible boundary on the propagation of gaseous detonations, in: Symposium (International) on Combustion, Vol. 10, Elsevier, 1965, pp. 817-830.
[15] J. A. Fay, Two-dimensional gaseous detonations: Velocity deficit, Physics of Fluids (1958-1988) 2 (3) (1959) 283-289.

[16] S. Tsuge, H. Furukawa, M. Matsukawa, T. Nakagawa, On the dual property and the limit of hydrogen-oxygen free detonation waves, Astronaut. Acta 15 (5) (1970) 377-386.

[17] W. W. Wood, J. G. Kirkwood, Diameter effect in condensed explosives. the relation between velocity and radius of curvature of the detonation wave, The Journal of Chemical Physics 22 (11) (1954) 1920-1924.

[18] M. Sichel, A hydrodynamic theory for the propagation of gaseous detonations through charges of finite width., AIAA Journal 4 (2) (1966) 264272.

[19] J. H. Lee, Dynamic Parameters of Gaseous Detonations, Annual Review of Fluid Mechanics 16 (1) (1984) 311-336.

[20] J. Shepherd, Detonation in gases, Proceedings of the Combustion Institute 32 (1) (2009) $83-98$.

[21] V. N. Gamezo, D. Desbordes, E. S. Oran, Formation and evolution of two-dimensional cellular detonations, Combustion and Flame 116 (1-2) (1999) $154-165$.

[22] E. S. Oran, J. W. Weber Jr, E. I. Stefaniw, M. H. Lefebvre, J. D. Anderson Jr, A numerical study of a two-dimensional H2-O2-Ar detonation using a detailed chemical reaction model, Combustion and Flame 113 (12) (1998) 147-163.

[23] A. K. Oppenheim, R. I. Soloukin, Experiments in gasdynamics of explosions, Annual Review of Fluid Mechanics 5 (1973) 31-58.

[24] M. I. Radulescu, G. J. Sharpe, C. K. Law, J. H. S. Lee, The hydrodynamic structure of unstable cellular detonations, Journal of Fluid Mechanics 580 (2007) 31-81.

[25] G. Ciccarelli, S. Dorofeev, Flame acceleration and transition to detonation in ducts, Progress in Energy and Combustion Science 34 (4) (2008) 499 -550 .

[26] M. I. Radulescu, J. H. Lee, The failure mechanism of gaseous detonations: experiments in porous wall tubes, Combustion and Flame 131 (1-2) (2002) $29-46$

[27] D. H. Edwards, G. O. Thomas, M. A. Nettleton, The diffraction of a planar detonation wave at an abrupt area change, Journal of Fluid Mechanics 95 (1979) 79-96.

[28] R. R. Nourgaliev, S. Y. Sushchikh, T. N. Dinh, T. G. Theofanous, Shock wave refraction patters at interfaces, International Journal of Multiphase Flow 31 (9) (2005) 969-995.

[29] D. Kessler, V. Gamezo, E. Oran, Simulations of flame acceleration and deflagration-to-detonation transitions in methane-air systems, Combustion and Flame 157 (11) (2010) 2063 - 2077.

[30] G. Goodwin, R. Houim, E. Oran, Effect of decreasing blockage ratio on DDT in small channels with obstacles, Combustion and Flame 173 (2016) $16-26$.

[31] G. Goodwin, R. Houim, E. Oran, Shock transition to detonation in channels with obstacles, Proceedings of the Combustion Institute (2016) doi:http://dx.doi.org/10.1016/j.proci.2016.06.160.

[32] R. W. Houim, A. Ozgen, E. S. Oran, The role of spontaneous waves in the deflagration-to-detonation transition in submillimetre channels, Combustion Theory and Modelling 20 (6) (2016) 1068-1087.

[33] R. W. Houim, K. K. Kuo, A low-dissipation and time-accurate method for compressible multi-component flow with variable specific heat ratios, Journal of Computational Physics 230 (23) (2011) 8527-8553.

[34] A. Harten, P. D. Lax, B. v. Leer, On upstream differencing and godunovtype schemes for hyperbolic conservation laws, SIAM review 25 (1) (1983) 35-61.

[35] E. F. Toro, M. Spruce, W. Speares, Restoration of the contact surface in the hll-riemann solver, Shock Waves 4 (1) (1994) 25-34.

[36] R. J. Spiteri, S. J. Ruuth, A new class of optimal high-order strongstability-preserving time discretization methods, SIAM Journal on $\mathrm{Nu}-$ merical Analysis 40 (2) (2003) 469-491.

[37] Center for Computational Sciences and Engineering, University of California, Berkeley, Boxlib (Retrieved 03-2015). URL https://ccse.lbl.gov/index.html

[38] B. Taylor, R. Houim, D. Kessler, V. Gamezo, E. Oran, Detonation initiation and shock-flame interaction in hydrogen-air mixtures, in: 51st AIAA Aerospace Sciences Meeting including the New Horizons Forum and Aerospace Exposition, 2013.

[39] M. Latini, O. Schilling, W. S. Don, Effects of weno flux reconstruction order and spatial resolution on reshocked two-dimensional richtmyer- 

805-836.

[40] M.-H. Wu, W.-C. Kuo, Accelerative expansion and DDT of stoichiometric ethylene/oxygen flame rings in micro-gaps, Proceedings of the Combustion Institute 34 (2) (2013) 2017 - 2024.

[41] D. Ranjan, J. Oakley, R. Bonazza, Shock-Bubble Interactions, Annual Review of Fluid Mechanics 43 (2011) 117-140.

[42] H. Hornung, Regular and mach reflection of shock waves, Annual Review of Fluid Mechanics 18 (1986) 33-58.

[43] G. Ben-Dor, Shock Wave Reflection Phenomena, Springer, 2008.

[44] R. Sorin, R. Zitoun, B. Khasainov, D. Desbordes, Detonation diffraction through different geometries, Shock Waves 19 (1) (2008) 11-23.

[45] L. J. Brown, G. Ravichandran, Analysis of oblique shock waves in solids using shock polars, Shock Waves 24 (4) (2013) 403-413.

[46] M. S. Ivanov, G. Ben-Dor, T. Elperin, A. N. Kudryavtsev, D. V. Khotyanovsky, The reflection of asymmetric shock waves in steady flows: a numerical investigation, Journal of Fluid Mechanics 469 (2002) 71-87.

[47] Y. Mahmoudi, K. Mazaheri, High resolution numerical simulation of triple point collision and origin of unburned gas pockets in turbulent detonations, Acta Astronautica 115 (2015) $40-51$.

[48] D. A. Schwer, K. Kailasanath, Effect of low pressure ratio on exhaust plumes of rotating detonation engines, in: $50^{\text {th }}$ AIAA/ASME/SAE/ASEE Joint Propulsion Conference and Exhibit, Cleveland, OH, 2014.

[49] E. S. Oran, Understanding explosions-From catastrophic accidents to creation of the universe, Proceedings of the Combustion Institute 35 (1) (2015) $1-35$.

[50] B. Taylor, D. Kessler, V. Gamezo, E. Oran, Numerical simulations of hydrogen detonations with detailed chemical kinetics, Proceedings of the Combustion Institute 34 (2) (2013) 2009 - 2016. 